\title{
Does fructose have a protective role on migraine?-experimental evidence in a rat model of metabolic syndrome under omega-3 supplementation
}

\author{
Isadora R. Barbosa ${ }^{1,2}$, Gabriela da Cunha ${ }^{2,3}$, Rodrigo B. M. Silva ${ }^{2}$, Raquel D. S. Freitas ${ }^{2}$, \\ Ana P. A. Dagnino ${ }^{2}$, Maria M. Campos ${ }^{1,2,3}$
}

${ }^{1}$ PUCRS, Programa de Pós-Graduação em Biologia Celular e Molecular, Escola de Ciências da Saúde e da Vida, Porto Alegre, RS, Brasil; ${ }^{2}$ PUCRS, Centro de Pesquisa em Toxicologia e Farmacologia, Escola de Ciências da Saúde e da Vida, Porto Alegre, RS, Brasil; ${ }^{3}$ PUCRS, Programa de Graduação em Odontologia, Escola de Ciências da Saúde e da Vida, Porto Alegre, RS, Brasil

Contributions: (I) Conception and design: IR Barbosa, MM Campos; (II) Administrative support: MM Campos; (III) Provision of study materials: MM Campos; (IV) Collection and assembly of data: IR Barbosa, G da Cunha, APA Dagnino, RBM Silva, RDS Freitas; (V) Data analysis and interpretation: IR Barbosa, RBM Silva, RDS Freitas, MM Campos; (VI) Manuscript writing: All authors; (VII) Final approval of manuscript: All authors.

Correspondence to: Maria M. Campos. Escola de Ciências da Saúde e da Vida, Porto Alegre, RS, Brasil. Email: maria.campos@pucrs.br; camposmmartha@yahoo.com.

\begin{abstract}
Background: Migraine is a highly disabling disease, for which current therapies are limited to symptom alleviation. There is compelling evidence linking migraine with metabolic disorders, but the causal relationship is not clear. Omega-3 (n-3) fatty acids have anti-inflammatory properties, with clear benefits in metabolic disorders, but its effects on migraine remains to be tested. We hypothesized that fructose-induced metabolic syndrome could aggravate migraine by increasing neuroinflammation and that n-3 treatment could mitigate it.
\end{abstract}

Methods: Male Wistar rats were used. Animals that received 10\% high fructose diet (HFD) or tap water were subdivided into two additional groups: with or without n-3 supplementation. Fifteen days before euthanasia, each group was subdivided into two additional groups: with or without nitroglycerin (NTG)induced migraine.

Results: HFD lessened the migraine-like painful symptoms, as indicated by decreased grimace scores, which paralleled with reduced CGRP and leptin serum levels, increased hypothalamic CGRP, and decreased hypothalamic adiponectin and IL-6. There was a recovery of body and adipose tissue weight, besides a reduction of crown-like structures (CLS) in the inguinal adipose tissue. N-3 supplementation had no effect on NTG-related pain, but it decreased body and adipose tissue weight of HFD and tap water NTG-injected rats. N-3 improved NTG-related affective behavior and inflammatory parameters in tap water NTGinjected rats, with decreased hypothalamic TNF, serum CGRP and inguinal adipose-tissue CLS.

Conclusions: HFD relieved NTG-induced pain, possibly due to decreased energy expenditure, minimizing migraine energy needs. N-3 exhibited favorable effects regarding affective behavior and central and peripheral inflammation, irrespective of HFD.

Keywords: Migraine; metabolic syndrome; fructose; omega-3 (n-3); nitroglycerin

Submitted Oct 26, 2021. Accepted for publication Jan 28, 2022.

doi: $10.21037 /$ atm-21-5699

View this article at: https://dx.doi.org/10.21037/atm-21-5699 


\section{Introduction}

Migraine has been considered the main cause of years living with disability, according to the 2019 Global Burden of Disease data, while analyzing subjects in economically active ages, from 15 to 49 years old (1). Its treatment is often challenging, due to the mild efficacy and the adverse effects of the currently available drugs. Migraine therapies are mostly limited to the alleviation of headache episodes and associated symptoms, leaving aside the biochemical changes inherent to the disease pathophysiology, allowing new migraine attacks (2).

The metabolic syndrome (MS) encompasses elevated triglycerides, high low-density lipoprotein (LDL) and low high-density lipoprotein (HDL) cholesterol levels, hyperinsulinemia, hyperglycemia, hypertension, and/or increased waist circumference, being related to lifestyle features, such as sedentarism, stress, and poor dietary habits. This assembly of alterations can favor the development of type 2 diabetes mellitus (T2DM), also increasing the risk of cardiovascular burden. The growing number of people living with MS worldwide poses a real challenge for the health systems, as for the direct and indirect costs and morbidity rates (3).

It has been demonstrated that omega-3 (n-3) polyunsaturated fatty acids display pain relief ensuing their anti-inflammatory/pro-resolution effects (4). Hence, n-3 might well represent an alternative for migraine control, with fewer side effects than the commonly used drug therapies (5). Furthermore, n-3 has clear benefits for metabolic disorders (6), permitting us to suggest that its application would be particularly interesting for individuals with MS plus migraine. There are some pieces of evidence linking migraine with metabolic disorders, such as MS and T2DM $(7,8)$. Notably, it has been suggested that migraineurs with aura presentation are at higher risk of developing MS, with an association with low HDL cholesterol levels plus hyperglycemia and abdominal obesity (9). However, the causal relationship concerning these conditions remains uncertain. It is worth noting that some authors have proposed that disrupted adipose tissue function in MS might increase cytokine and adipokine production, leading to a proinflammatory state, which in turn prompts neuroinflammation, finally aggravating headache bouts (10).

Considering the link between migraine and MS, besides the promising effects of $n-3$ in both conditions, the present study was designed to investigate whether the induction of
MS, by long-term fructose intake, might alter nitroglycerin (NTG)-evoked migraine attacks in rats, also evaluating the effects of $n-3$ supplementation in this context. For this purpose, we analyzed anthropometric data, adipose tissue dysregulation, biochemical and inflammatory profiling, painful-like changes, as well as affective behavioral alterations, when the experimental paradigms of migraine and MS were combined, with or without $n-3$ administration. Our data shed new light on the intricate connection between migraine and MS, further extending the previous evidence on the effects of $n-3$ in either disease state. We present the following article in accordance with the ARRIVE reporting checklist (available at https://atm.amegroups.com/ article/view/10.21037/atm-21-5699/rc) (11).

\section{Methods}

\section{Animals}

Male Wistar rats (6-week-old, total $\mathrm{N}=96$ animals) weighing 170 to $240 \mathrm{~g}$ at the onset of experiments, were obtained from the Central Animal House of the Pontifícia Universidade Católica do Rio Grande do Sul (CeMBE; PUCRS; Brazil). The age of animals was selected based on a previous literature study (12). Males were used instead of females, considering the great hormonal influence in the migraine NTG model (13). From a total of 96 rats, three died due to NTG injections and one was euthanized after oral gavage of $n-3$, by recommendation of the responsible Veterinarian. Therefore, a final number of 92 animals was included in the analysis. The animals were housed under standard conditions of temperature $\left(22 \pm 2{ }^{\circ} \mathrm{C}\right)$, light $(12-\mathrm{h}$ light-dark cycle) and humidity (50-70\%), in ventilated cages, with autoclaved wood chip bedding. They received a standard rat chow diet $\left(\mathrm{Nuvilab}^{\circledR}\right)$, with free access to filtered water or $10 \%$ fructose solution, depending on the experimental group. The experimental protocols followed the current Brazilian guidelines for the care and use of animals for scientific and didactic procedures, from the National Council for the Control of Animal Experimentation (CONCEA, Brazil). Experiments were performed under a project license (9088) granted by Institutional Animal Care and Use Committee of PUCRS on December 5, 2018. The number of animals was based on previous studies (14) with an alpha of $5 \%$ and statistical power of $80 \%$ (GraphPad StatMate 2.0) which resulted in a number of 12 rats per group. 


\section{Induction of MS and experimental groups}

MS was induced as described previously (15). Rats received $10 \%$-fructose solution in the drinking water for eight weeks. The control animals received filtered water during the same period. Rats were weighed $(\mathrm{g})$ three times a week, and the chow was weighed $(\mathrm{g})$ every day. The water was refilled, the consumed volume $(\mathrm{mL})$ was measured every day, and the results by cage were pooled weekly. The glucose levels $(\mathrm{mg} / \mathrm{dL})$ were measured at the end of experiments with a digital glucometer (Accu-Check III, Boehringer Mannheim, Germany). After four weeks, the animals submitted to high fructose diet (HFD; $\mathrm{N}=12$ ) or normal diet (water; $\mathrm{N}=12$ ) were randomly subdivided into additional experimental groups: with $\mathrm{n}-3$ supplementation ( $\mathrm{n}-3 ; \mathrm{N}=12$ each); without $\mathrm{n}-3$ supplementation (vehicle; $\mathrm{N}=12$ each). For randomization, the animals were initially allocated into two major groups, with or without HFD, after determining the mean body weight of each cage with four rats. The allocation of animals into the NTG and/ or $n-3$ groups was performed by alternating the cages in the following order, considering the body weigh means: (I) water + saline; (II) water + n-3; (III) water + NTG; (IV) water $+n-3+$ NTG, and the same was carried out for HFD groups. NTG-injected rats were maintained separated from control animals. The general experimental design and group distribution are depicted in Figure 1.

\section{Chronic migraine model}

Chronic migraine induction was accomplished as described previously (14). Nitroglycerin (NTG; Broilo Pharmaceutical products, Brazil) $-5 \mathrm{mg} / \mathrm{mL}$-dissolved in $30 \%$ alcohol, $30 \%$ propylene glycol and water were used. NTG was administered intraperitoneally (i.p.) with a sterile glass syringe, in a volume of $2 \mathrm{~mL} / \mathrm{kg}$, every three days, to reach the dose of $10 \mathrm{mg} / \mathrm{kg}$, totaling five NTG injections (Figure 1). The animals in the control group received saline solution $(2 \mathrm{~mL} / \mathrm{kg})$ i.p. at the same time intervals.

\section{Omega-3 (n-3) treatment}

The treatment with n-3 was initiated four weeks after the onset of HFD protocol. Half of animals received n-3 through fish oil administration (Essential Nutrition; Essentia Pharma, Brazil) composed of $55 \% \mathrm{n}-3$ (1 g contains $360 \mathrm{mg}$ EPA and $240 \mathrm{mg}$ DHA), by gavage, in a dose of $1 \mathrm{~g} / \mathrm{kg}$, every day, for four weeks, as previously described (16). After two weeks of treatment with n-3, the protocol of migraine induction with NTG injections was initiated. Control animals received saline solution at the same schedule of treatment.

\section{Behavioral assays}

Animals were transferred to the testing room $40 \mathrm{~min}$ prior to the behavioral tests. grimace scale, photophobia evaluation in the light/dark box, and anxiety-like behavior assessment in the elevated plus maze (EPM) were carried out at $30 \mathrm{~min}, 60 \mathrm{~min}$ or $24 \mathrm{~h}$ after the NTG injection, respectively. Two experimenters conducted all of the behavioral tests to avoid biased outcomes. The protocols for each assay are described below.

\section{Rat grimace scale}

After each NTG injection, rats were placed in a transparent glass cage and recorded for $60 \mathrm{~min}$. After each test, the device was thoroughly cleaned with ethanol $70 \%$. Following the experiment, the records were assessed for scoring by one trained research assistant who was blinded to the experimental condition. After $30 \mathrm{~min}$ of recording, 10 pictures were taken every $3 \mathrm{~min}$ and then the images were scored for three features, namely orbital tightening, nose/cheek flattening, and ear changes using a scale of $0-2$ (not present, moderate, obvious). The scores were averaged across the 10 pictures to produce an overall pain score for each rat.

\section{Photophobia assessment in the light/dark box}

After 60 min of each NTG injection, migraine-related photophobia was assessed in a light/dark box (Insight, Brazil), which consisted of a two-chamber apparatus, with dimensions of $210 \mathrm{~mm} \times 450 \mathrm{~mm} \times 410 \mathrm{~mm}$ for the bright side, and of $210 \mathrm{~mm} \times 350 \mathrm{~mm} \times 410 \mathrm{~mm}$ for the dark side. A red adhesive paper was taped over the acrylic lid of the black chamber to reduce illumination. The rats were placed in the corner of the white chamber facing away from the experimenter and were allowed to explore the two chambers for $20 \mathrm{~min}$. The time spent in the white chamber during this period was measured and expressed in seconds. The results of the five NTG injections were summed up. After each test, the device was thoroughly cleaned with $30 \%$ ethanol.

\section{Assessment of affective behavior}

After $24 \mathrm{~h}$ of each NTG injection, the elevated plus maze (EPM) paradigm was used for assessment of affectiveemotional disorders related to pain. The animals were 


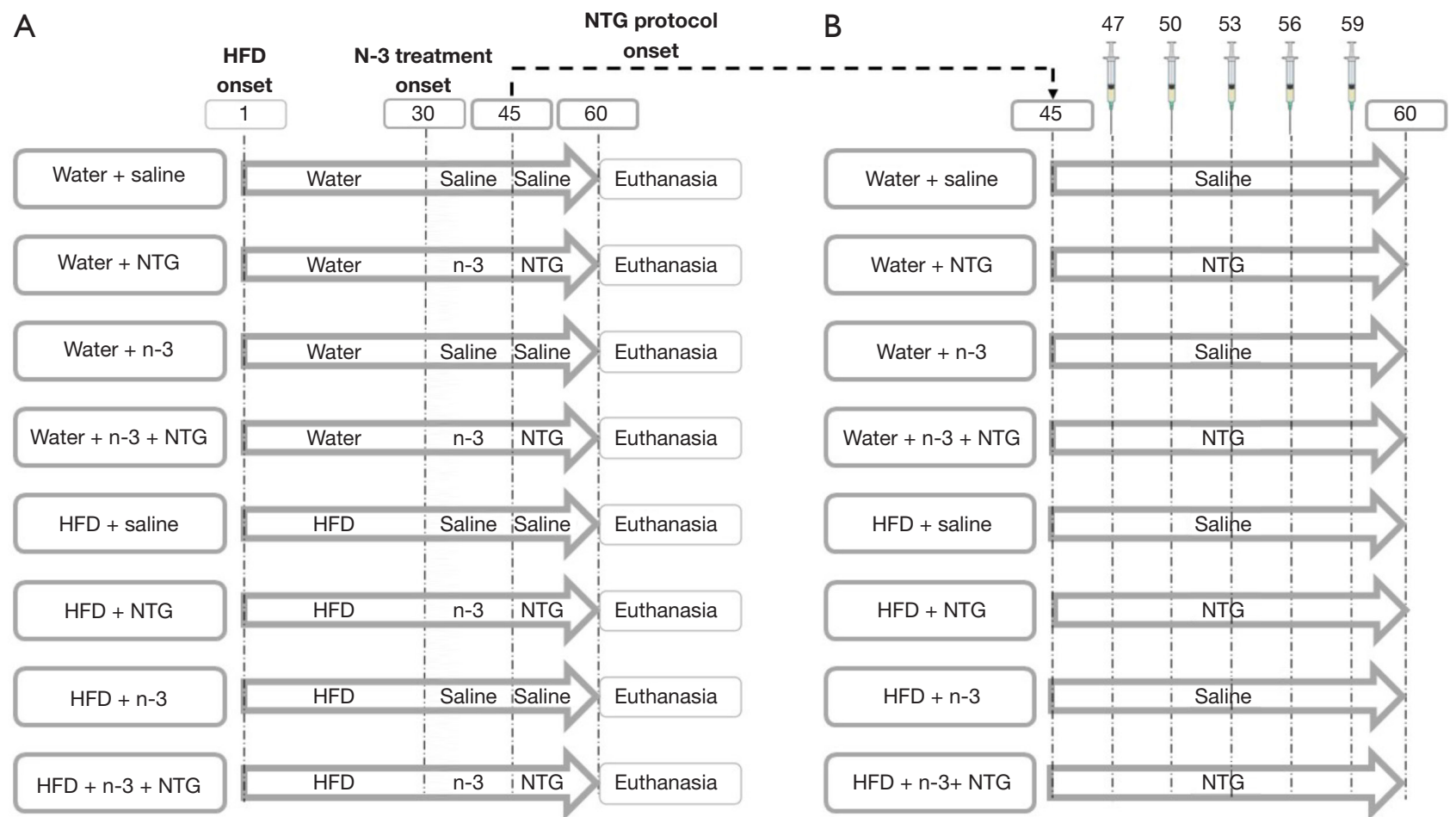

C

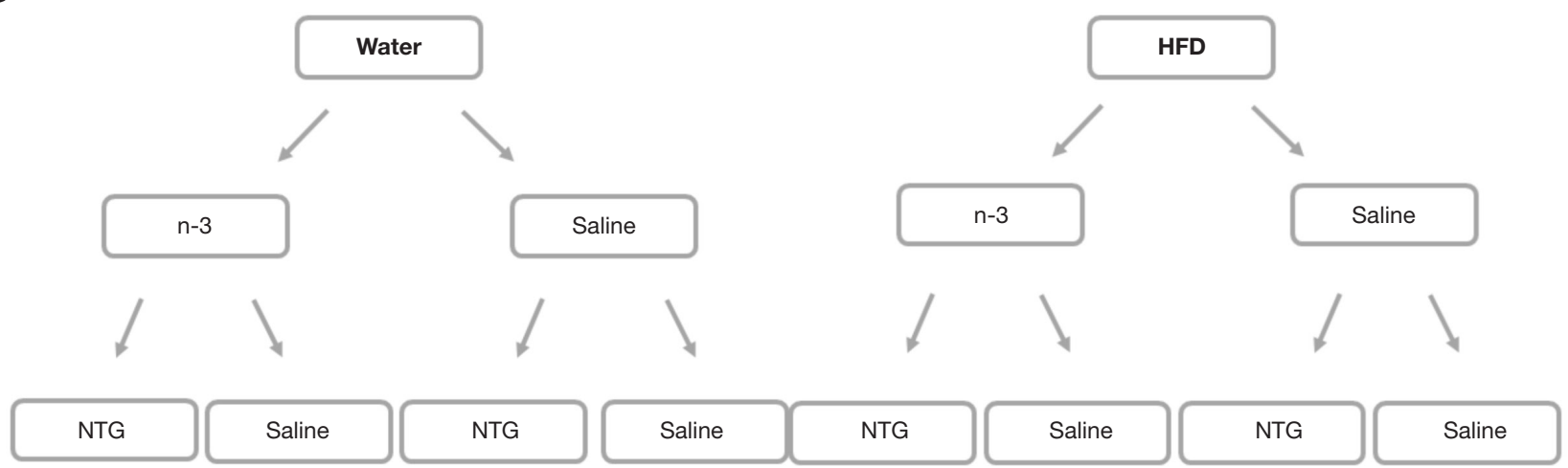

Figure 1 General experimental design. (A) The MS protocol started on day one and finished on day 60; the n-3 supplementation protocol started on day 30 and ended on day 60; the migraine induction lasted from days 45 to 60 . The animals were assessed in behavioral tasks and submitted to euthanasia for sample collection. (B) The chronic migraine protocol comprised five i.p. injections of NTG at days 47, 50, 53, 56, and 59; control animals received vehicle at the same treatment schedule. (C) Scheme showing the group distribution. Rats were initially distributed into two experimental groups that received tap water or $10 \%$ fructose solution for 60 days. After 30 days of supplementation onset, animals were randomly subdivided into control or n-3-treated groups. Additional subgroups were formed after 15 days of treatment: one-half of animals were subjected to the protocol of migraine induction, by five NTG i.p. injections every three days, whereas control animals received vehicle by the same route of administration. MS, metabolic syndrome; NTG, nitroglycerin; HFD, high fructose diet.

placed in the center of the maze facing an open arm and were allowed to explore the apparatus for five minutes. The time spent as well as the entries in the open and closed arms were registered during this period. An entry into an open arm was defined as all four paws crossing the center of the maze. During the permanence in the open arm, the head dipping counts were also registered. After each test, the device was thoroughly cleaned with ethanol $70 \%$. 


\section{Levels of cytokines and adipokines}

Animals were euthanized 8 weeks after the onset of the HFD consumption by deep anesthesia with isoflurane. Immediately after euthanasia, the blood was collected by cardiac puncture. After a resting period for coagulation, the samples were centrifuged $\left(4,500 \mathrm{rpm}, 12 \mathrm{~min}, 4^{\circ} \mathrm{C}\right)$, and the serum was aliquoted and stored at $-80{ }^{\circ} \mathrm{C}$ until the analysis (up to six months at most). Serum and hypothalamus were collected to evaluate the levels of CGRP, tumor necrosis factor (TNF), interleukin-6 (IL-6), leptin, and adiponectin. Cytokines and adipokines were analyzed by sandwich ELISA using DuoSet ${ }^{\circledR}$ kits according to the manufacturer's instructions (R\&D Systems, Minneapolis, MN, USA) and the results were expressed in picograms per $\mathrm{mL}$ The kits and the limits of detection were: rat TNF- $\alpha$ (DY510; 62.5 to $4,000 \mathrm{pg} / \mathrm{mL}$ ); rat IL-6 (DY506; 62.5 to $4,000 \mathrm{pg} / \mathrm{mL}$ ); rat leptin (MOB00B; 62.50 to $4,000 \mathrm{pg} / \mathrm{mL}$ ) and rat adiponectin (DY3100-05; 156.0 to $10,000 \mathrm{pg} / \mathrm{mL}$ ). The experiments with serum samples were run in duplicate, and hypothalamic samples were run in simplicate.

\section{Lipid profile}

The serum levels of triglycerides (catalog No. 87), total cholesterol catalog No. 76), HDL (catalog No. 13) and non-HDL cholesterol fractions were determined by using colorimetric enzyme assay kits, according to the manufacturer's instructions (LabTest, Brazil) and results were expressed in milligrams per $\mathrm{dL}$.

\section{Histopathological analysis}

Inguinal (subcutaneous) and epidydimal (visceral) white adipose tissues (WAT) were collected and fixed in $10 \%$ formaldehyde solution for further histopathological analysis. WAT samples were included in paraffin and sectioned in 5 - $\mu \mathrm{m}$ slices, for staining with hematoxylin and eosin, as described elsewhere (17). Briefly, the slides were stained for $30 \mathrm{~s}$ in hematoxylin, followed by $30 \mathrm{~s}$ in eosin, under agitation, intercalated with water washes. At the end of staining procedures, the slides were submitted to dehydration steps with alcohol and xylene. Images were captured using a Zeiss AxioImager M2 light microscope under $\times 200$ magnification (Carl Zeiss, Gottingen, Germany) and were analyzed using NIH ImageJ $1.36 \mathrm{~b}$ Software. Adipocyte areas were measured manually, using the free-hand function, after adjusting the image scale in square micrometer. The frequency size of adipocyte areas was determined by a trained experimenter blinded to the groups. Crown-like structures (CLS) density was obtained by counting the total number of CLS in each image of inguinal and epidydimal WAT samples.

\section{Statistical analysis}

The results are expressed as the mean \pm the standard error mean. Brown-Forsythe and Kolmogorov-Smirnov tests were used for checking data normality. Column data (group as the only factor) was analyzed by one-way ANOVA (parametric data) or Kruskal-Wallis (non-parametric data) tests. For grouped results (with treatment and time as factors), data was evaluated by two-way ANOVA (parametric data) or mixed-effect analysis (non-parametric data). When the interaction of factors was statistically significant ( $\mathrm{P}$ values less than 0.05$)$, pairwise comparisons were conducted by using uncorrected Fisher's LSD's or Dunn's post-hoc tests, after one-way ANOVA and Kruskal Wallis, respectively. For two-way ANOVA and mixedeffect analysis, pairwise comparisons were conducted by using Tukey or uncorrected Fisher's LSD's post-hoc tests, respectively (GraphPad Software Inc., San Diego, CA, USA, version 8.0.2). $\mathrm{P}$ values less than 0.05 were considered statistically significant. The variations in the experimental $\mathrm{N}$ due to sample limitations are described in each legend figure.

\section{Results}

\section{Food and liquid intake at different time points}

We examined the chronic effects of $10-\%$ fructose supplementation and NTG injections on food and liquid intake in rats. HFD rats showed a decrease in food intake over 60 days of experiments (Figure 2A,2B). NTG-injected rats also presented a significant reduction of food intake in comparison with the respective control group, this feature being observed on days 50 to 56,59 and 60 . NTGinjected rats under HFD showed suppressed feeding only on days 45 and 58, compared with their HFD counterparts. Conversely, NTG-injected rats receiving tap water consumed more food than HFD NTG-injected rats on days 45 to 47 (Figure 2B). Analyzing the AUC throughout the protocol of chronic migraine induction (from days 45 to 60 ), it is possible to observe that NTG injections reduced food intake, regardless of HFD or tap water consumption. 

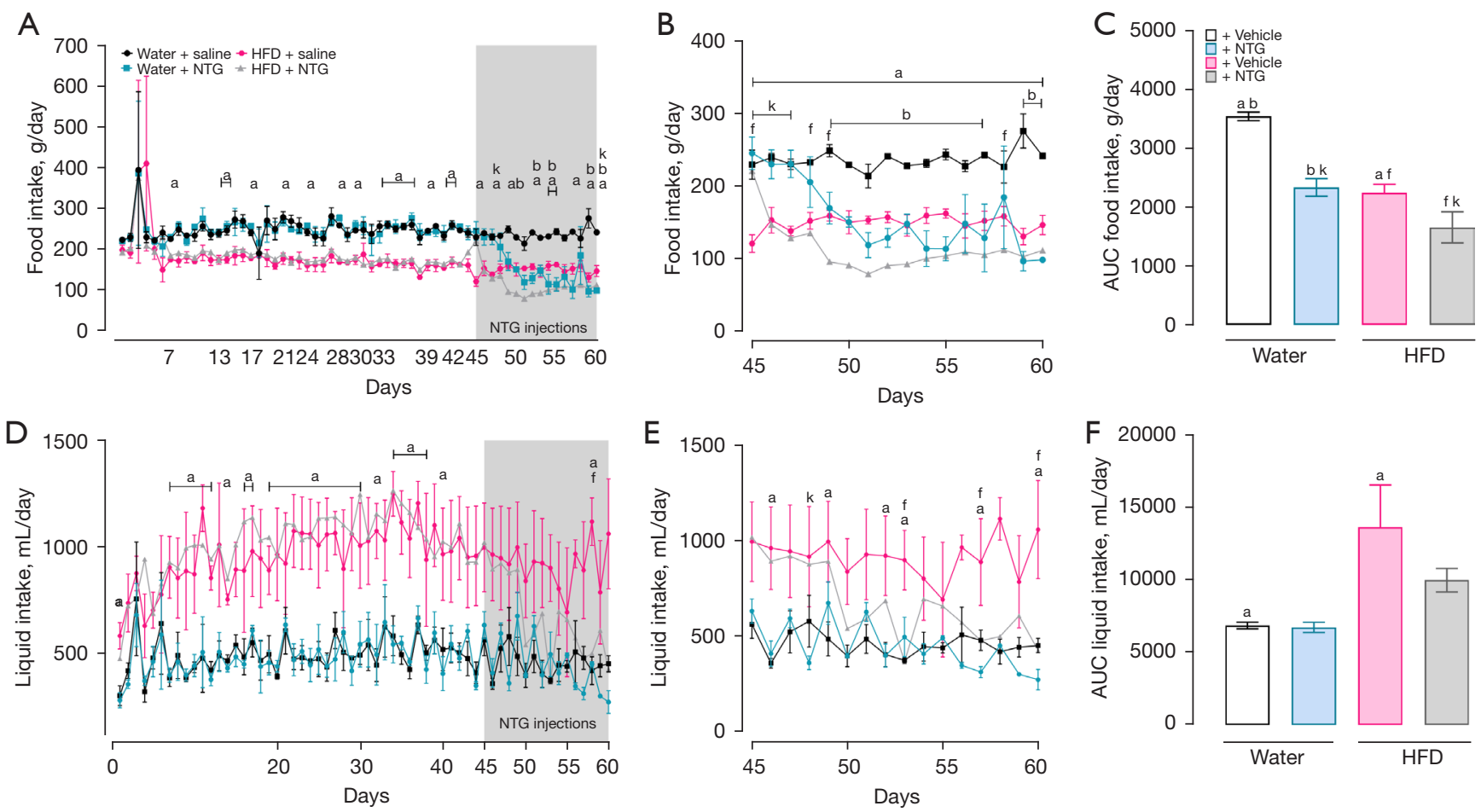

Figure 2 Food and liquid intake of rats in the water control group (water + saline), HFD control group (HFD + saline), NTG-injected rats (water + NTG), and HFD NTG-injected rats (HFD + NTG). (A) Food intake until day 60, before and after the migraine induction protocol. (B) Food intake from days 45 to 60, during migraine induction protocol. (C) The area under the curve of food intake from days 45 to 60. (D) Liquid intake until day 60, before and after migraine induction protocol. (E) Liquid intake from days 45 to 60 during migraine induction protocol. (F) The area under the curve of liquid intake from days 45 to 60 . Data are presented as mean \pm SEM of 10 to 12 rats per group (two-way ANOVA followed by Tukey's post hoc test; mixed-effect analysis followed by uncorrected Fisher's LSD's post hoc test; or one-way ANOVA followed by uncorrected Fisher's LSD's post hoc). ${ }^{\text {a }}, \mathrm{P}<0.05$ water + saline $v s$. HFD + saline; ${ }^{\text {b }}, \mathrm{P}<0.05$ water + saline vs. water + NTG; ${ }^{\mathrm{f}}, \mathrm{P}<0.05 \mathrm{HFD}+$ saline $v$ s. HFD + NTG; ${ }^{\mathrm{k}}, \mathrm{P}<0.05$ water + NTG vs. HFD + NTG. NTG, nitroglycerin; HFD, high fructose diet; SEM, standard error of the mean.

Importantly, HFD NTG-injected rats significantly reduced chow consumption when compared with NTGinjected rats receiving tap water (Figure 2C). Concerning the liquid intake, HFD increased the consumption over 60 days of evaluation (Figure 2D). Throughout the migraine induction protocol, NTG injections lessened the liquid intake in the HFD group, but not in rats drinking tap water. HFD NTG-injected rats drank more liquids on day 48 in relation to NTG-injected rats taking tap water (Figure 2E). Scrutinizing the AUC of liquid intake during the protocol of migraine induction, NTG injections neither affected this behavior in rats receiving tap water nor in rats submitted to HFD; however, HFD increased the liquid intake compared with control rats receiving tap water (Figure $2 F$ ).

\section{Body weight changes}

We analyzed the body weight means and the body weight variation by subtracting the initial body weight from the final body weight at different time points: from days 1 to 30 , from days 30 to 45 , from days 45 to 60 , and from days 1 to 60 (Table 1). From days 1 to 30, the body weight means were not significantly different between HFD and tap water groups. Conversely, at the same time point, HFD rats showed a significant increase in body weight variation when compared with tap water rats. There was no significant effect for n-3 treatment in either body weight means or body weight variation, from days 30 to 45 . HFD rats treated with $n-3$ had increased body weight means and variation in comparison with $\mathrm{n}$-3-treated tap water rats. A 
Table 1 Body weight and body weight variation from days 1 to 30, 30 to 45 and 45 to 60 in water control group (water + saline), HFD control group rats (HFD + saline), NTG-injected rats (water + NTG), and HFD NTG-injected rats (HFD + NTG), with or without n-3 supplementation (water + n-3; HFD + n-3; n-3 + NTG; HFD + n-3 + NTG)

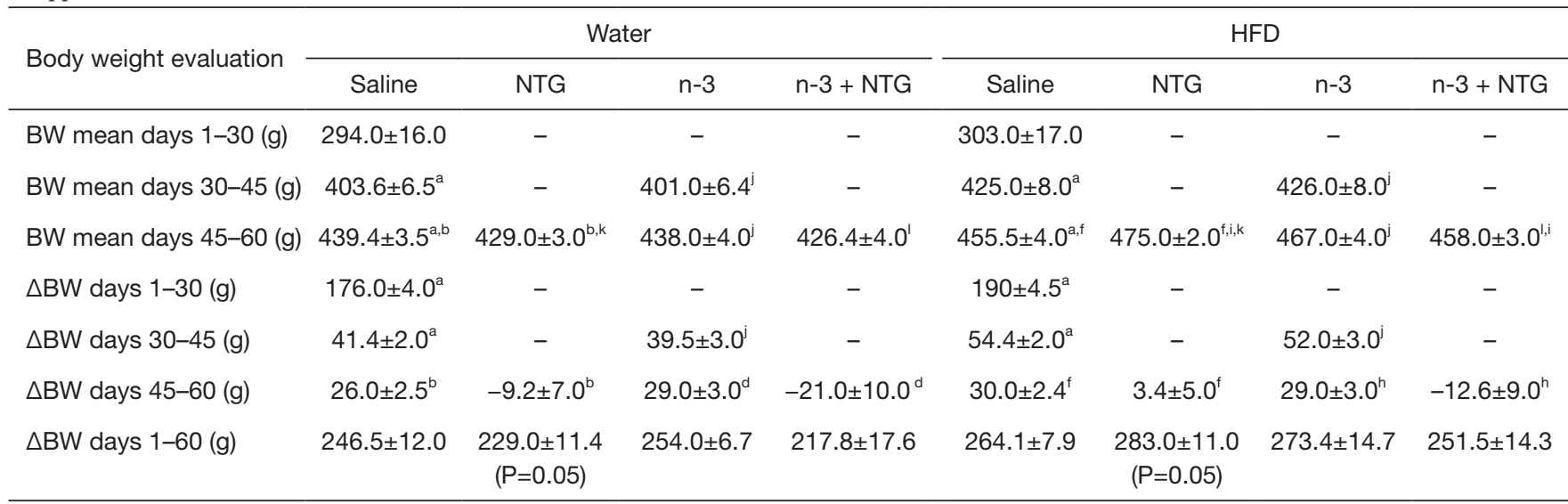

Data are presented as means \pm SEM of 10 to 12 rats per group for body weight (two-way ANOVA followed by Tukey's post hoc test; oneway ANOVA followed by uncorrected Fisher's LSD's post hoc; or Kruskal-Wallis followed by uncorrected Dunn's post hoc test). ${ }^{2}$, $\mathrm{P}<0.05$ water + saline vs. HFD + saline; ${ }^{b}, \mathrm{P}<0.05$ water + saline vs. water + NTG; ${ }^{d}, \mathrm{P}<0.05$ water + n-3 vs. water + n-3 + NTG; ${ }^{f}, \mathrm{P}<0.05 \mathrm{HFD}+$ saline vs. HFD + NTG; ${ }^{\mathrm{h}}, \mathrm{P}<0.05$ HFD + n-3 vs. HFD + n-3 + NTG; ${ }^{i}, \mathrm{P}<0.05$ HFD + NTG vs. HFD + n-3+ NTG; ${ }^{j}, \mathrm{P}<0.05$ water $+\mathrm{n}-3$ vs. HFD + n-3; ${ }^{k}, \mathrm{P}<0.05$ water + NTG vs. HFD + NTG; ', P<0.05 water + n-3 + NTG vs. HFD + n-3+ NTG. BW, body weight; NTG, nitroglycerin; HFD, high fructose diet; SEM, standard error of the mean.

similar effect was observed for $\mathrm{n}$-3-free HFD rats, while compared with n-3 free tap water rats. From days 45 to 60 , HFD rats displayed increased body weight means than tap water rats, with no difference for body weight variation. Additionally, n-3 treated HFD rats displayed increased body weight mean in relation to $\mathrm{n}-3$ treated tap water rats. NTG-injected tap water rats had their body weight mean and variation significantly decreased compared with the tap water control group, unlike HFD NTG-injected rats, which presented an increased body weight mean compared with their NTG-free counterparts. HFD NTGinjected rats had an increased body weight mean than tap water NTG-injected rats, while body weight variation was not statistically different between these groups. N-3 supplementation restored the body weight mean of NTGinjected rats under HFD. The body weight variation of n-3-treated HFD rats was significantly different from the n-3-treated HFD NTG-injected group, indicating that n-3 treatment decreased the body weight along with NTG injections. From days 1 to 60 , the body weight variation of HFD NTG-injected rats was slightly higher $(\mathrm{P}=0.05)$ than that from tap water NTG-injected animals (Table 1).

\section{Inguinal and epididymal WAT dynamics}

We tested whether the supplementation with $10 \%$ fructose solution or migraine induction by NTG injections altered the subcutaneous (inguinal) and visceral (epididymal) WAT dynamics and whether n-3 treatment changed these parameters. Tap water NTG-injected rats showed a partial decrease of inguinal WAT weight $(\mathrm{P}=0.069)$, compared with their NTG-free counterparts, with no significant difference for HFD NTG-injected rats. Interestingly, HFD NTGinjected rats exhibited a significant increase of inguinal WAT weight when compared with tap water NTG-injected rats, a difference that was also seen with $n-3$ treatment. The $\mathrm{n}-3$ supplementation did not alter inguinal WAT weight in tap water rats compared with their counterparts. Conversely, n-3-treated HFD rats exhibited significantly higher inguinal WAT weight, when compared with n-3treated rats that received tap water (Figure $3 A$ ).

We also evaluated the inguinal adipocyte areas and the size-frequency distribution in the different experimental groups. As expected, the mean inguinal adipocyte area of HFD rats was significantly higher when compared with tap water rats. HFD NTG-injected rats showed a trend to decrease of the mean inguinal adipocyte area $(\mathrm{P}=0.07)$ in relation to the HFD control group, whereas the tap water NTG-injected rats did not display any differences, when compared with the tap water control group. N-3treated HFD rats showed a slight decrease of inguinal adipocyte areas $(\mathrm{P}=0.06)$ compared with their $\mathrm{n}-3$-free 
A

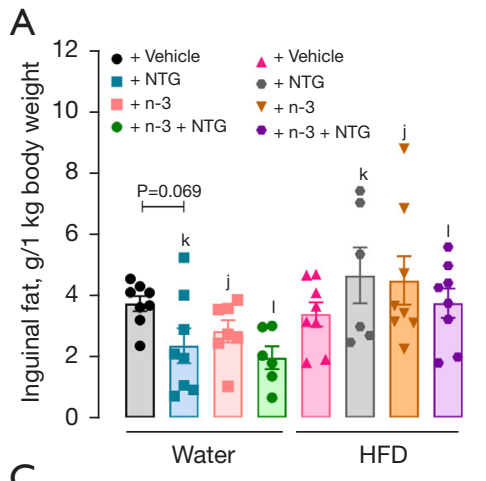

\section{$c$}

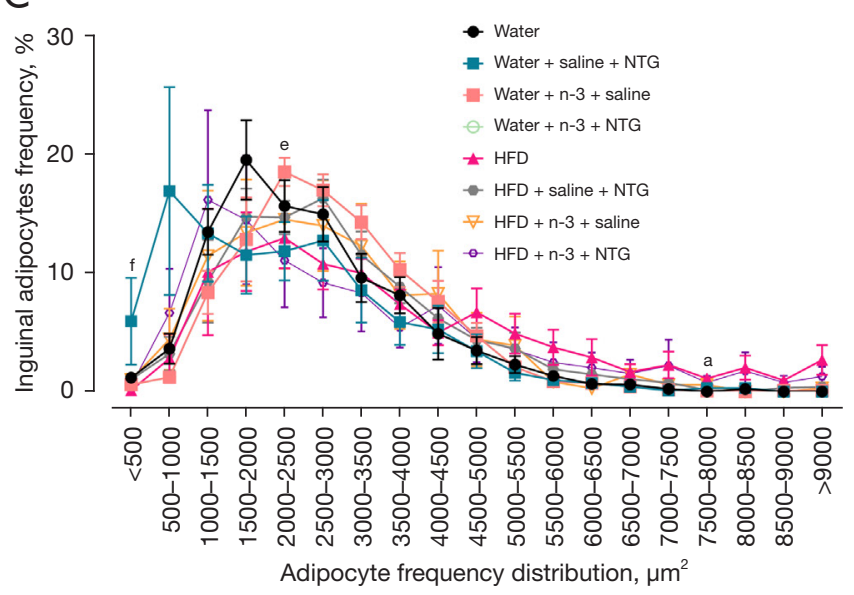

B
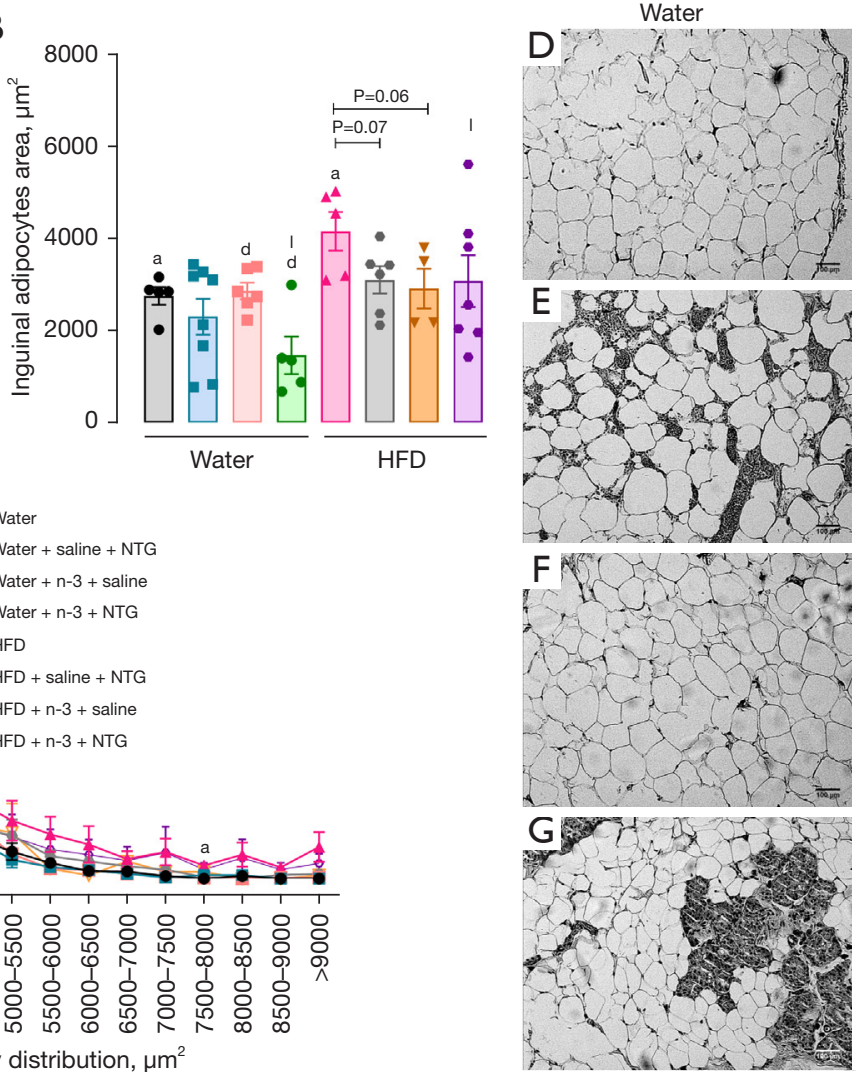
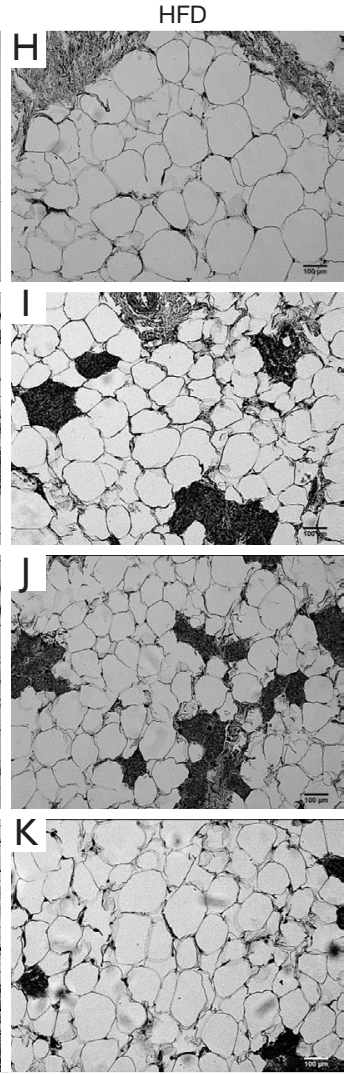

Figure 3 Profile of inguinal white adipose tissue in the different experimental groups. (A) Inguinal white adipose tissue weight; (B,C) histological analysis of inguinal adipocyte size and frequency of inguinal adipocyte sizes, after migraine and HFD induction protocols. The evaluations were performed by an examiner blinded to the experimental groups. After the analysis, one representative picture per group was selected to compose the panel of representative images. (D-K) Images were captured using a Zeiss AxioImager M2 light microscope under ×200 magnification (Carl Zeiss, Gottingen, Germany). Representative histological images of inguinal adipose tissue in: water control group (water + saline; D), NTG-injected rats (water + NTG; E), rats with n-3 supplementation (water + n-3; F), NTG-injected rats with n-3 supplementation (water + n-3 + NTG; G), HFD control group (HFD + saline; H), HFD NTG-injected rats (HFD + NTG; I), HFD rats with n-3 supplementation (HFD + n-3; J), and HFD NTG-injected rats with n-3 supplementation (HFD + n-3 + NTG; K), respectively. Data are presented as mean \pm SEM of 4 to 8 rats per group (mixed-effect analysis followed by uncorrected Fisher's LSD's post hoc test; or one-way ANOVA followed by uncorrected Fisher's LSD's post hoc). ${ }^{a}, \mathrm{P}<0.05$ water + saline $v s$. HFD + saline; ${ }^{\mathrm{d}}, \mathrm{P}<0.05$ water $+\mathrm{n}-3$ vs. water + n-3 + NTG; ${ }^{\mathrm{e}}, \mathrm{P}<0.05$ water + NTG vs. water + n-3 + NTG; ${ }^{\mathrm{f}}, \mathrm{P}<0.05$ HFD + saline vs. HFD + NTG; ${ }^{\mathrm{i}}, \mathrm{P}<0.05 \mathrm{HFD}+\mathrm{NTG} v s$. HFD + n-3+ NTG; ${ }^{j}, \mathrm{P}<0.05$ water + n-3 vs. HFD + n-3; ${ }^{k}, \mathrm{P}<0.05$ water + NTG vs. HFD + NTG. NTG, nitroglycerin; HFD, high fructose diet; SEM, standard error of the mean.

counterparts, with no differences for the tap water group. Lastly, n-3-treated tap water NTG-injected rats showed a reduced mean inguinal adipocyte area than the NTGfree rats (Figure 3B). As for the inguinal adipocyte sizefrequency distribution, HFD NTG-injected rats displayed a significantly higher number of adipocytes with an area of $<500 \mu^{2}$ than HFD NTG-free rats. N-3-treated tap water NTG-injected rats showed decreased adipocyte areas, with a higher number of adipocytes displaying an area ranging from 2,000 to 2,500 $\mathrm{\mu m}^{2}$, compared with n-3-free tap water NTG-injected rats (Figure 3C). Representative images of inguinal adipocytes are provided in panels Figure $3 \mathrm{D}-3 \mathrm{~K}$.

Concerning the epididymal WAT weight, NTGinjected rats presented a significant reduction of this parameter compared with the respective NTG-free control groups that had received either tap water or HFD. Moreover, n-3-treated HFD NTG-injected rats showed higher epididymal adiposity than n-3 treated and NTG- 

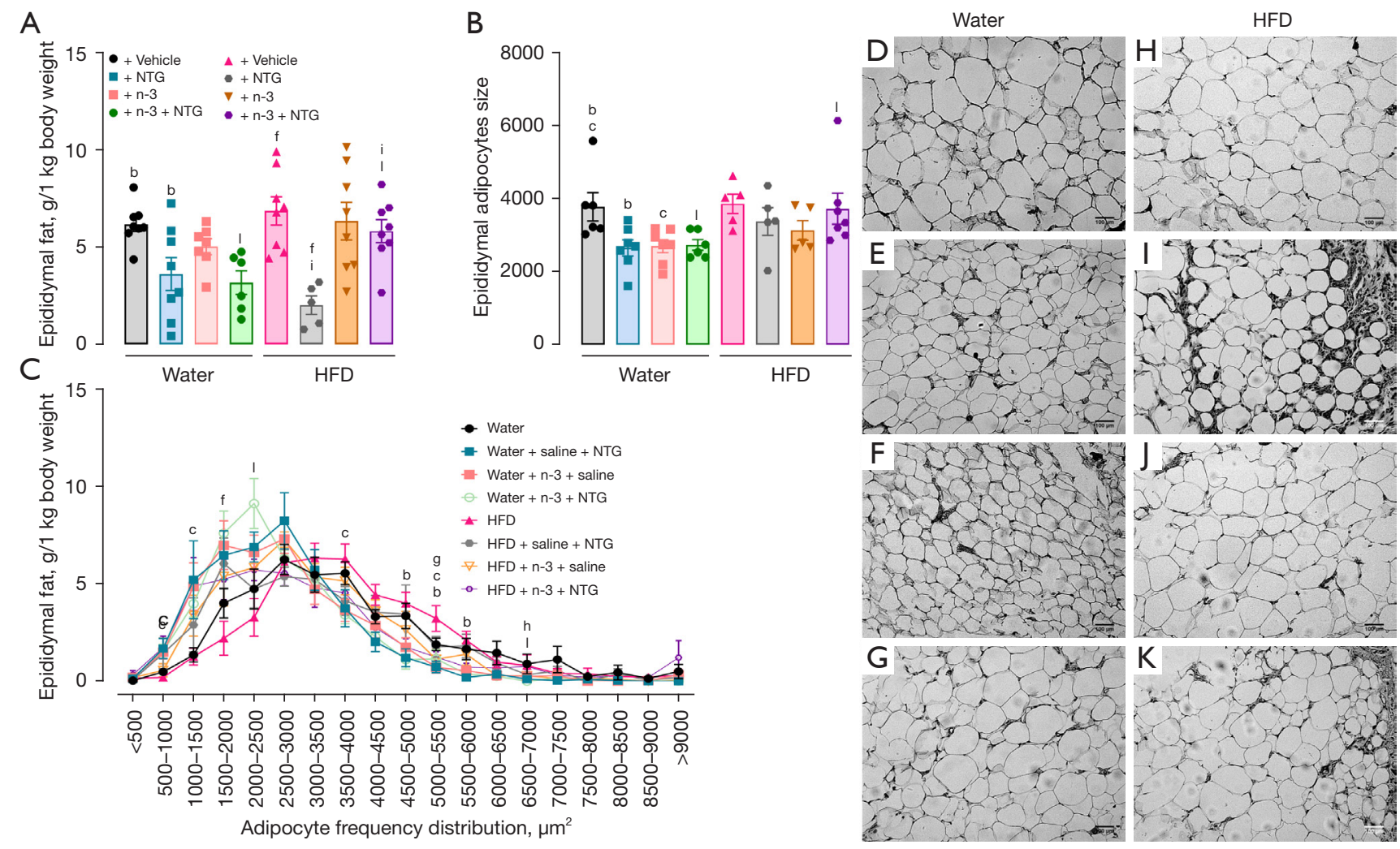

Figure 4 Profile of epididymal white adipose tissue in the different experimental groups. (A) Epididymal white adipose tissue weight; $(B, C)$ histological analysis of epididymal adipocyte sizes and frequency of epididymal adipocyte sizes after migraine and HFD induction protocols. The evaluations were performed by an examiner blinded to the experimental groups. After the analysis, one representative picture per group was selected to compose the panel of representative images. (D-K) Images were captured using a Zeiss AxioImager M2 light microscope under×200 magnification (Carl Zeiss, Gottingen, Germany). Representative histological images of epididymal adipose tissue in: water control group (water + saline; D), NTG-injected rats (water + NTG; E), rats with n-3 supplementation (water + n-3; F), NTGinjected rats with n-3 supplementation (water + n-3 + NTG; G), HFD control group (HFD + saline; H), HFD NTG-injected rats (HFD + NTG; I), HFD rats with n-3 supplementation (HFD + n-3; J), and HFD NTG-injected rats with n-3 supplementation (HFD + n-3 + NTG; K), respectively. Data are presented as mean \pm SEM of 4 to 8 rats per group (mixed-effect analysis followed by uncorrected Fisher's LSD's post hoc test; or one-way ANOVA followed by uncorrected Fisher's LSD's post hoc test). ${ }^{\text {b }}, \mathrm{P}<0.05$ water + saline vs. water + NTG; ${ }^{\mathrm{c}}, \mathrm{P}<0.05$ water + saline $v s$. water $+\mathrm{n}-3 ;{ }^{\mathrm{f}}, \mathrm{P}<0.05 \mathrm{HFD}+$ saline $v s$. HFD + NTG; ${ }^{\mathrm{g}}, \mathrm{P}<0.05 \mathrm{HFD}+\mathrm{saline} v s . \mathrm{HFD}+\mathrm{n}-3 ;{ }^{\mathrm{h}}, \mathrm{P}<0.05 \mathrm{HFD}$ + n-3 vs. HFD + n-3 + NTG; ${ }^{i}, \mathrm{P}<0.05$ HFD + NTG vs. HFD + n-3+ NTG; ${ }^{1}, \mathrm{P}<0.05$ water + n-3 + NTG vs. HFD + n-3 + NTG. NTG, nitroglycerin; HFD, high fructose diet; SEM, standard error of the mean.

injected tap water animals, as well as than HFD NTGinjected rats without n-3 treatment (Figure 4A). Extending this data, tap water NTG-injected rats exhibited a decrease of epididymal adipocyte areas, in relation to the NTG-free control group, with no significant differences for the HFD group. Curiously, the n-3 treated tap water rats displayed decreased epididymal adipocyte area compared with the n-3-free control group; there were no changes for the HFD groups. Also, n-3 treated HFD NTG-injected rats showed increased epididymal adipocyte areas, compared with their n-3 treated tap water counterparts (Figure $4 B$ ). Both the NTG-injected HFD and the NTG-injected tap water rats showed reduced epididymal adipocyte size frequencies compared with their NTG-free control rats; with the smallest adipocytes for the NTG-injected HFD rats ranging from 1,500 to $2,000 \mathrm{\mu m}^{2}$, whereas the most adipocytes of control water group ranged from 4,500 to $6,000 \mu \mathrm{m}^{2}$. Remarkably, tap water rats treated with $n-3$ showed a decrease of epididymal adipocyte size frequencies, in comparison with their $n-3$ free counterparts, the former 
displaying a higher number of adipocytes with areas ranging from 5,000 to $5,500 \mathrm{~m}^{2}$, compared with their $\mathrm{n}-3$ treated counterparts. Also, n-3 treated HFD rats showed a decrease of epididymal adipocyte size frequencies, as the n-3-free HFD rats displayed a higher number of adipocytes with areas ranging from 5,000 to 5,500, compared with their HFD n-3-free counterparts. N-3-treated HFD NTG-injected rats showed increased epididymal adipocyte size frequencies when compared with tap water rats with the same treatment; the former displaying a higher number of adipocytes with areas ranging from 6,500 to $7,000 \mathrm{\mu m}^{2}$ (Figure $4 C$ ). Representative images of epididymal adipocytes are provided in panels Figure $4 D-4 K$.

We carried out a semi-quantitative analysis of crownlike structures (CLS) in epididymal and inguinal rat adipose tissues (Figure 5). Tap water NTG-injected rats displayed a higher intensity of CLS in visceral and subcutaneous WAT, than tap water control rats, with a higher occurrence of CLS in the subcutaneous WAT. Interestingly, this effect was almost totally reversed by $\mathrm{n}-3$ supplementation in the tap water rats. HFD increased the subcutaneous CLS compared with rats receiving tap water. N-3 treatment switched this parameter as well. Remarkably, HFD NTG-injected rats showed CLS only in the visceral WAT, with a less intensity than HFD NTGfree rats. Unexpectedly, n-3 treated HFD NTG-injected rats displayed increased CLS in visceral and subcutaneous WAT in comparison with $\mathrm{n}$-3-free HFD NTG-injected rats. Lastly, CLS were superior in the WAT of tap water NTG-injected rats than in the WAT of HFD NTGinjected rats, both visceral and subcutaneous (Figure 5).

\section{Nociception endpoints}

We analyzed photophobia by using the light/dark box and the pain-like symptoms by the grimace scale evaluation. NTG injections significantly decreased the time spent in the light chamber of the light/dark box in all groups (Figure $6 \mathrm{~A}$ ). The AUC of grimace scores showed a pronounced pain-like behavior in groups that received NTG. Curiously, HFD NTG-injected rats displayed a significant decrease of grimace scores, when compared with tap water NTG-injected rats, an effect that has been prevented by n-3 supplementation (Figure 6B,6C). Representative grimace images taken 30 minutes after the last NTG injection are depicted in panels Figure 6D-6K. These conclusions are supported by descriptive data showing the minimal and maximal AUC values, besides the sum of values in each experimental group (Table S1).

\section{Changes in MS and migraine-like biomarkers}

NTG did not change the serum levels of CGRP, neither in the tap water nor in the HFD group, compared with their NTG-free counterparts. Nevertheless, n-3-treated tap water NTG-injected rats displayed decreased serum CGRP levels compared with their n-3-free and NTG-injected counterparts. HFD NTG-injected rats showed a slight decrease in the serum CGRP contents, when compared with tap water NTG-injected rats $(\mathrm{P}=0.06)$. No changes were observed for $\mathrm{n}$-3-treatment in the HFD groups (Figure $7 A$ ). N-3 treated tap water rats displayed decreased serum leptin compared with the n-3-free control rats. Either HFD or tap water NTG-injected rats showed a decrease in serum leptin levels when compared with their NTG-free counterparts. Interestingly, n-3 treated HFD rats exhibited higher serum leptin levels than the $\mathrm{n}-3$ treated HFD NTG-injected group. Also, n-3-treated HFD rats presented higher serum leptin contents than $n$-3-treated tap water rats (Figure $7 B$ ). As for the analysis of circulating adiponectin, HFD rats' levels were decreased in relation to tap water control rats. Also, the same occurred in rats receiving $n-3$, when compared with those without $n-3$ supplementation, both in the tap water or in the HFD rats. Furthermore, the n-3-treated NTG-injected rats displayed decreased serum adiponectin levels, while compared to n-3free NTG-injected rats, regardless of HFD. Also, there was a tendency toward decreased serum adiponectin levels in n-3 treated HFD rats compared with n-3 treated tap water rats (Figure $7 C$ ). There were no detectable TNF and IL-6 contents in serum of any evaluated group.

Relating to the hypothalamic parameters, HFD rats displayed decreased hypothalamic CGRP levels than tap water rats, while HFD NTG-injected rats showed increased hypothalamic CGRP than HFD control rats. No changes were seen for n-3 treated rats (Figure 8A). The n-3-treated tap water group displayed decreased hypothalamic IL6, when compared with $\mathrm{n}$-3-free tap water rats. A slight decrease in this parameter was observed for HFD rats, if compared with tap water rats $(\mathrm{P}=0.06)$. A slight increase $(\mathrm{P}=0.08)$ of hypothalamic IL-6 was seen in $\mathrm{n}-3$ treated tap water NTG-injected rats, when compared with n-3 treated NTG-free rats. Also, a mild increase $(\mathrm{P}=0.08)$ in this parameter was seen in n-3 treated HFD NTGinjected rats, compared with n-3-free HFD NTG-injected rats. Surprisingly, hypothalamic IL-6 had a slight decrease 

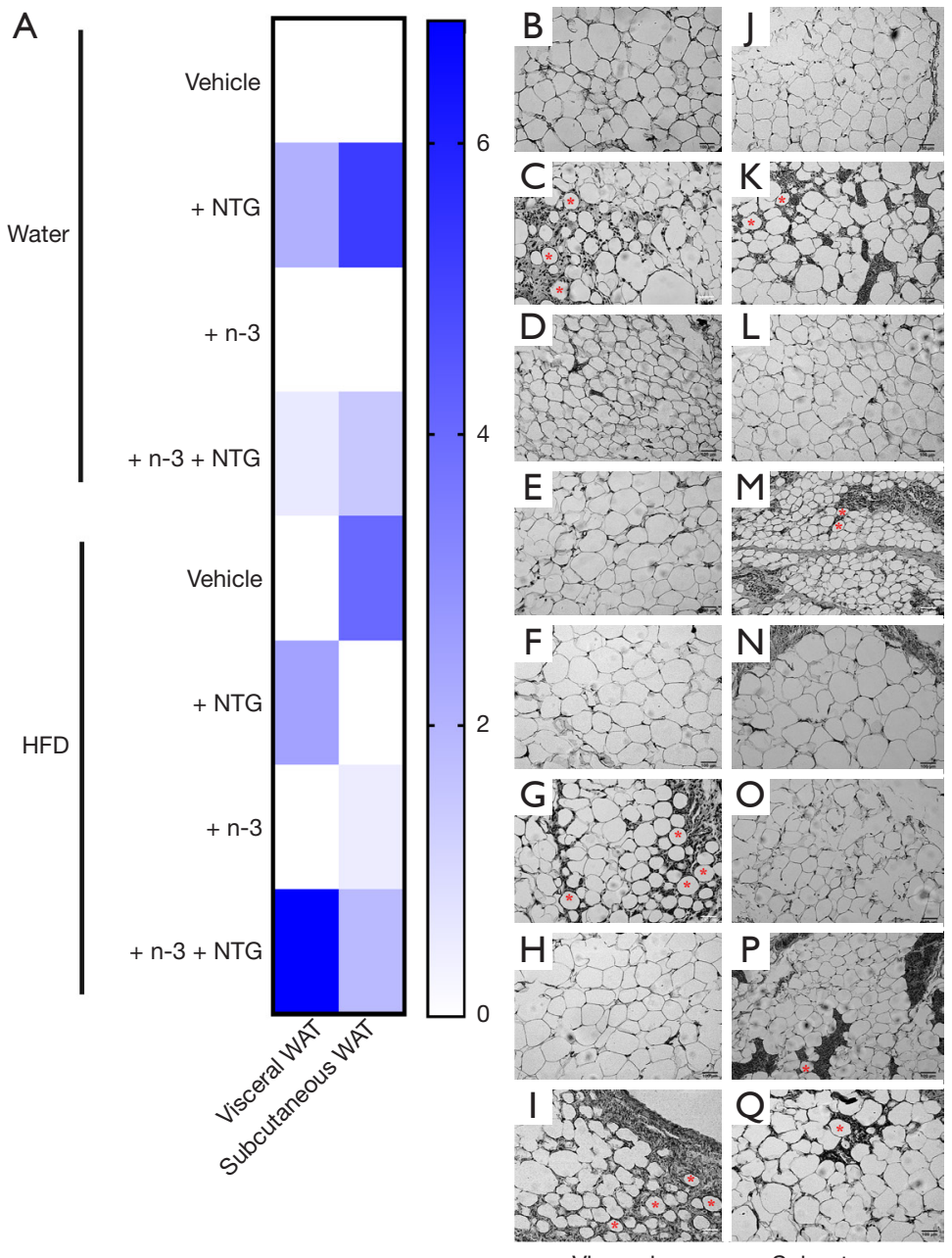

Figure 5 Heat mapping showing the semi-quantitative analysis of CLS in inguinal (subcutaneous WAT) and epididymal (visceral WAT) adipose tissue. (A) Colors vary in shades of blue, with an intensity proportional to the number of CLS in the respective adipose tissue. The evaluations were performed by an examiner blinded to the experimental groups. After the analysis, one representative picture per group was selected to compose the panel of representative images. Images were captured using a Zeiss AxioImager M2 light microscope under $\times 200$ magnification (Carl Zeiss, Gottingen, Germany). Representative histological images of inguinal adipose tissue in: water control group (water + saline; B), NTG-injected rats (water + NTG; C), rats with n-3 supplementation (water + n-3; D), NTG-injected rats with n-3 supplementation (water + n-3 + NTG; E), HFD control group (HFD + saline; F), HFD NTG-injected rats (HFD + NTG; G), HFD rats with n-3 supplementation (HFD + n-3; H), and HFD NTG-injected rats with n-3 supplementation (HFD + n-3 + NTG; I), respectively; and epididymal adipose tissue in: water control group (water + saline; J), NTG-injected rats (water + NTG; K), rats with n-3 supplementation (water + n-3; L), NTG-injected rats with n-3 supplementation (water + n-3 + NTG; M), HFD control group (HFD + saline; N), HFD NTG-injected rats (HFD + NTG; O), HFD rats with n-3 supplementation (HFD + n-3; P), and HFD NTG-injected rats with n-3 supplementation (HFD + n-3 + NTG; Q), respectively. The red asterisks indicate the adipocytes presenting CLS. CLS, crown-like structure. WAT, white adipose tissues; NTG, nitroglycerin; HFD, high fructose diet.

$(\mathrm{P}=0.08)$ in HFD NTG-injected rats compared with tap water NTG-injected rats (Figure 8B). As for hypothalamic TNF contents, there was a significant decrease in $n-3$ treated tap water NTG-injected rats, when compared with their n-3-free counterparts; no changes were observed in HFD groups (Figure 8C). Concerning hypothalamic leptin contents, n-3 treated HFD NTG-injected rats displayed a slight decrease compared with n-3-free HFD NTG- 


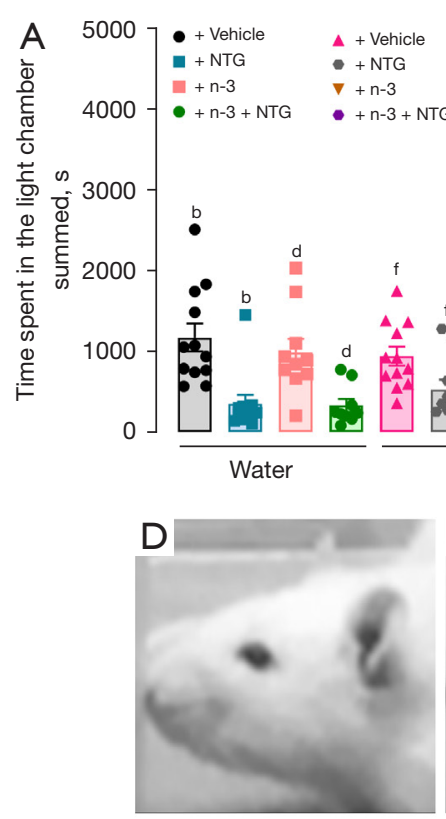

Water

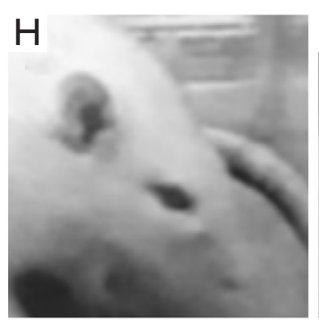

HFD

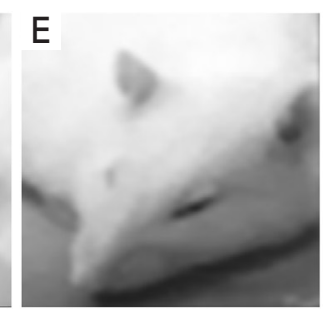

NTG

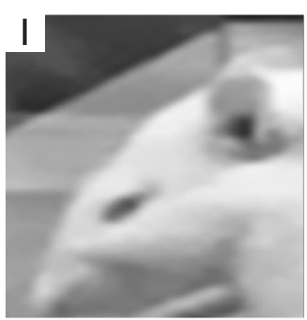

$\mathrm{HFD}+\mathrm{NTG}$
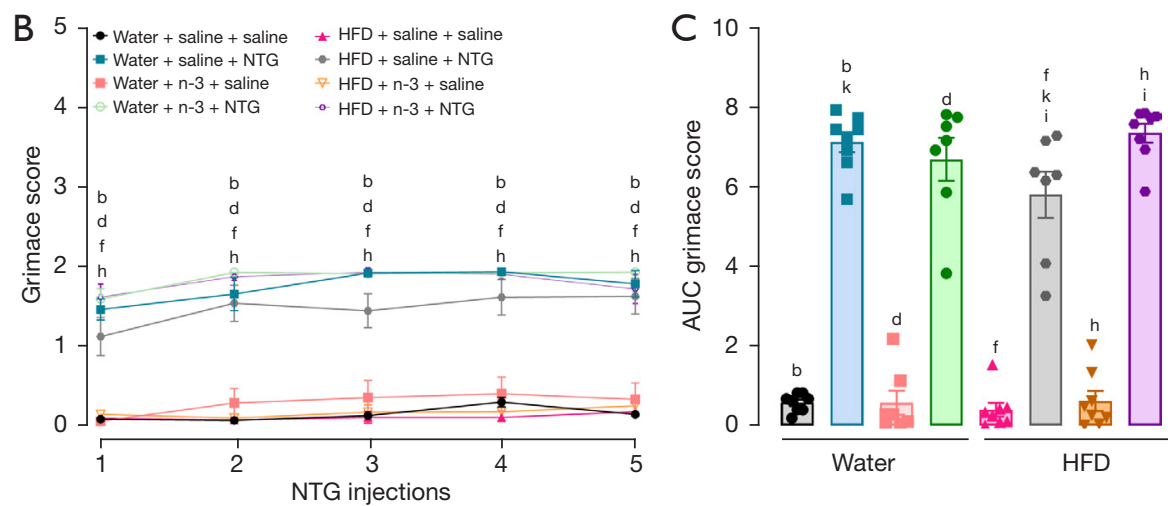

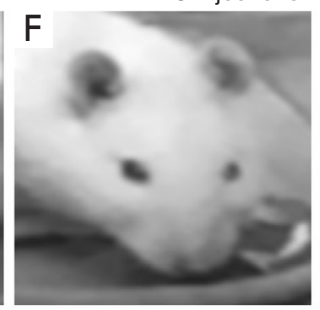

Water $+n-3$

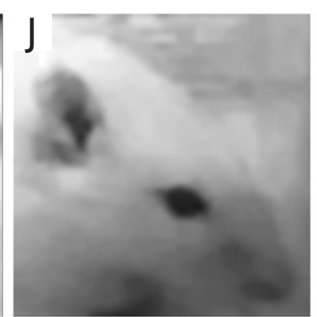

$H F D+n-3$

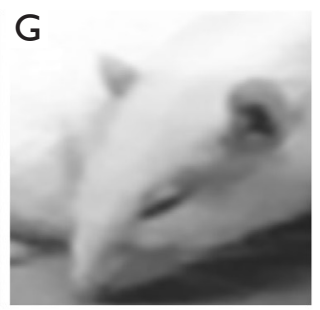

Water + n-3 + NTG

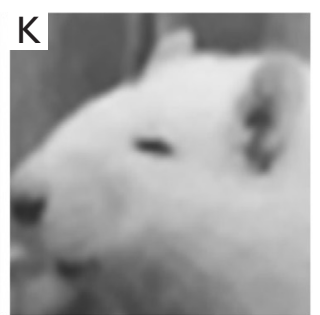

HFD + n-3 + NTG

Figure 6 Migraine-like symptoms in the different experimental groups. (A) Photophobia assessed by the sum of the time spent in the light chamber of a light-dark box, after one hour of NTG injections; (B) grimace scores assessed during one hour after each NTG injection; (C) area under the curve of grimace scores assessed during one hour after NTG injections. The grimace scale was evaluated by an examiner blinded to the experimental groups. After the analysis, one representative picture per group was selected to compose the panel of representative images, taken with a digital camera (Apple iPAD/8 MP camera). (D-L) Representative images taken 30 minutes after the last NTG injection in water control group (water + saline; D), NTG-injected rats (water + NTG; E), rats with n-3 supplementation (water + n-3; F), NTG-injected rats with n-3 supplementation (water + n-3 + NTG; G), HFD control group (HFD + saline; H), HFD NTG-injected rats (HFD + NTG; I), HFD rats with n-3 supplementation (HFD + n-3; J), and HFD NTG-injected rats with n-3 supplementation (HFD + n-3 + NTG; K), respectively. Data are presented as mean \pm SEM of 7 to 12 rats per group (Kruskal Wallis followed by uncorrected Dunn's post hoc test; mixed-effect analysis followed by uncorrected Fisher's LSD's post hoc test; or one-way ANOVA followed by uncorrected Fisher's LSD's post hoc test). ', $\mathrm{P}<0.05$ water + saline vs. water + NTG; ${ }^{\mathrm{d}}, \mathrm{P}<0.05$ water + n-3 vs. water + n-3 + NTG; ${ }^{\mathrm{f}}, \mathrm{P}<0.05 \mathrm{HFD}+$ saline $v s . \mathrm{HFD}+\mathrm{NTG} ;{ }^{\mathrm{h}}, \mathrm{P}<0.05 \mathrm{HFD}+\mathrm{n}-3$ vs. HFD + n-3 + NTG; ${ }^{k}, \mathrm{P}<0.05$ water + NTG vs. HFD + NTG; ${ }^{\mathrm{i}}, \mathrm{P}<0.05$ HFD + NTG vs. HFD + n-3 + NTG. NTG, nitroglycerin; HFD, high fructose diet; SEM, standard error of the mean.

injected rats $(\mathrm{P}=0.063)$, with no changes for tap water groups (Figure 8D). As for hypothalamic adiponectin levels, there was a decrease of this biomarker in the HFD NTG-injected rats, when compared with HFD NTG-free rats. Also, n-3 treated tap water NTG-injected rats displayed reduced adiponectin levels than their NTG-free counterparts (Figure 8E).

\section{Biochemical parameters}

At the end of the MS protocol, HFD rats displayed a mild increase in glycemia compared with tap water control rats $(\mathrm{P}=0.055)$. HFD NTG-injected rats presented decreased glycemia levels, when compared with their NTG-free equivalents. No differences were noticed between tap water 

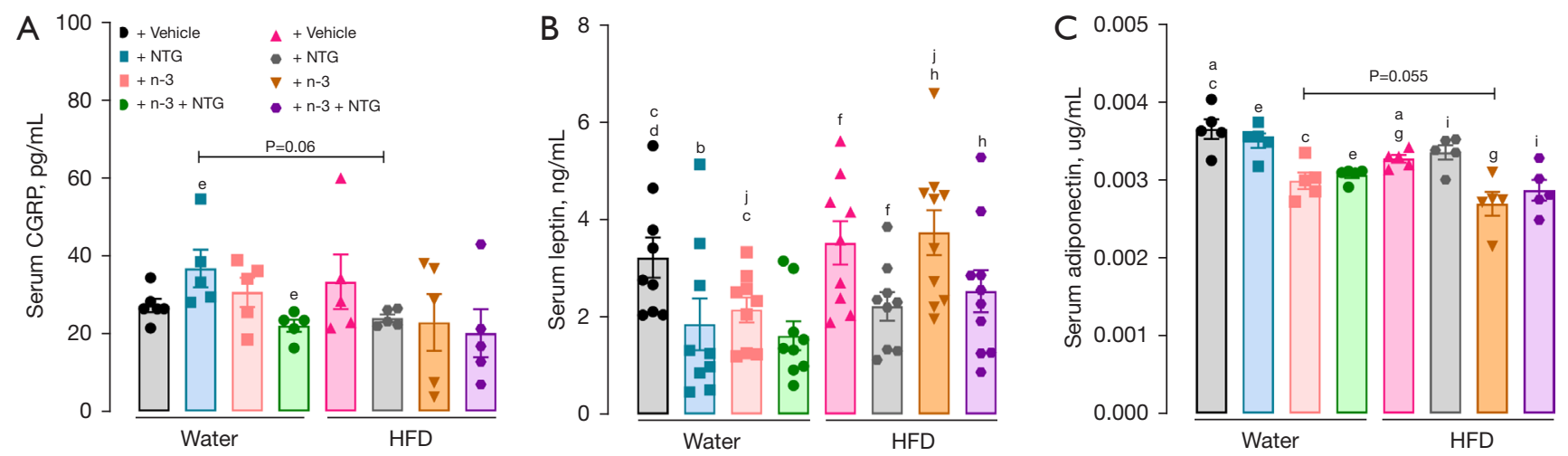

Figure 7 Effects of NTG, HFD, and n-3 treatment on serum levels of (A) CGRP, (B) leptin, and (C) adiponectin, measured by enzyme immunoassay at the end of experiments. Data are presented as means \pm SEM of 5 to 10 rats per group (one-way ANOVA followed by uncorrected Fisher's LSD's post hoc test). ${ }^{\mathrm{a}}, \mathrm{P}<0.05$ water $v s . \mathrm{HFD} ;{ }^{\mathrm{b}}, \mathrm{P}<0.05$ water + saline $v s$. water $+\mathrm{NTG} ;{ }^{\mathrm{c}}, \mathrm{P}<0.05$ water $v s$. water $+\mathrm{n}-3$; ${ }^{\mathrm{d}}$, $\mathrm{P}<0.05$ water + n-3 vs. water + n-3 + NTG; ${ }^{\mathrm{e}}$, water + NTG vs. water + n-3+ NTG; ${ }^{\mathrm{f}}, \mathrm{P}<0.05 \mathrm{HFD}+\mathrm{saline} v s . \mathrm{HFD}+\mathrm{NTG} ;{ }^{\mathrm{g}}, \mathrm{P}<0.05 \mathrm{HFD}$ vs. HFD + n-3; ${ }^{\mathrm{h}}, \mathrm{P}<0.05$ HFD + n-3 vs. HFD + n-3 + NTG; ${ }^{\mathrm{i}}, \mathrm{P}<0.05$ HFD + NTG vs. HFD + n-3 + NTG; ${ }^{\mathrm{j}}, \mathrm{P}<0.05$ water + n-3 vs. HFD $+\mathrm{n}-3$. NTG, nitroglycerin; HFD, high fructose diet; SEM, standard error of the mean.
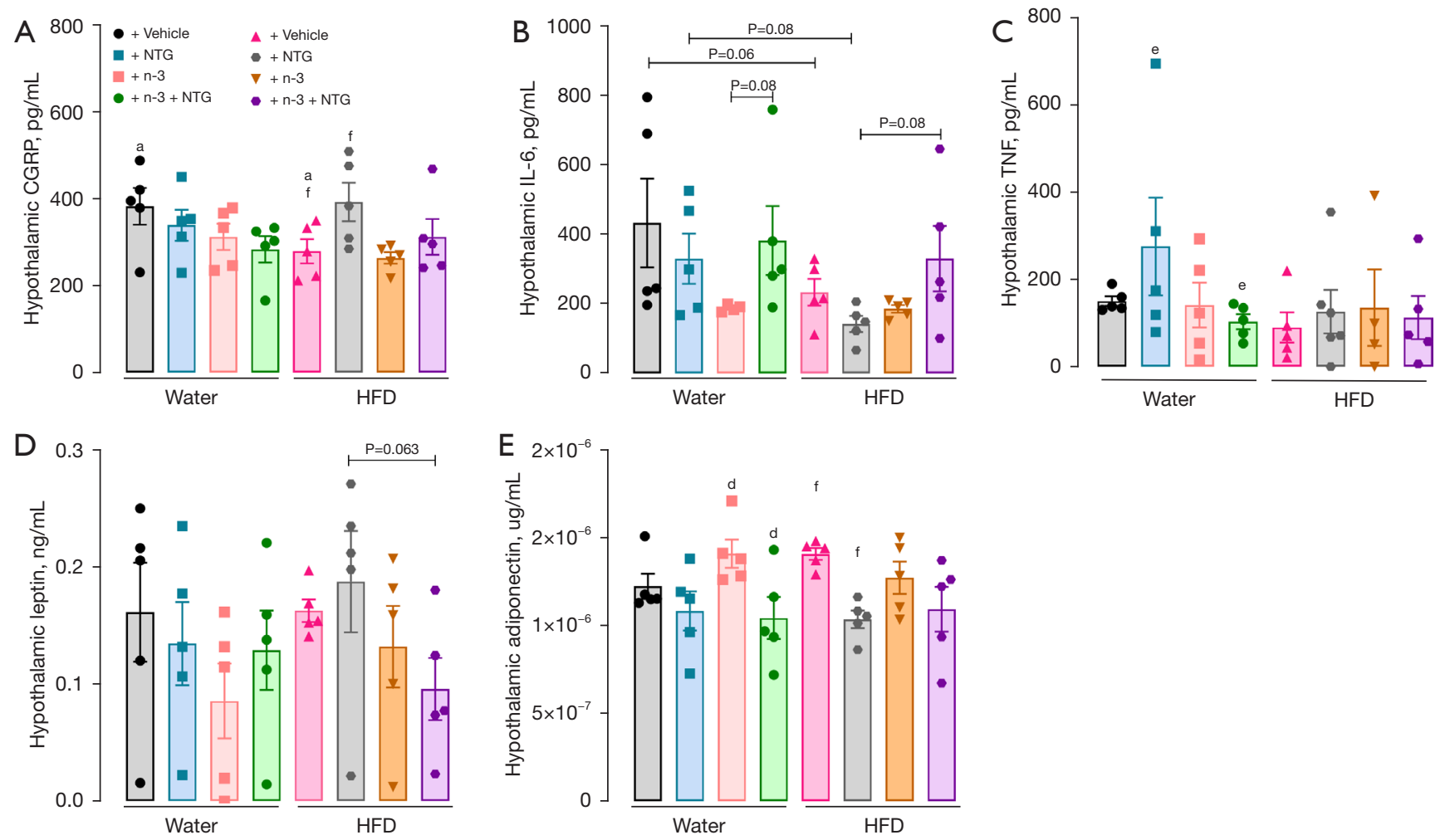

Figure 8 Effects of NTG, HFD, and n-3 treatment on hypothalamic levels of (A) CGRP, (B) IL-6, (C) TNF, (D) leptin, and (E) adiponectin, measured by enzyme immunoassay at the end of experiments. Data are presented as means \pm SEM of 4 to 6 rats per group (oneway ANOVA followed by uncorrected Fisher's LSD's post hoc test). ${ }^{a}, \mathrm{P}<0.05$ water + saline $v s . \mathrm{HFD}+\mathrm{saline}$; ${ }^{\mathrm{d}}, \mathrm{P}<0.05$ water $+\mathrm{n}-3$ vs. water + n-3 + NTG; ${ }^{\mathrm{e}}, \mathrm{P}<0.05$ water + NTG vs. water + n-3 + NTG; ${ }^{\mathrm{f}}, \mathrm{P}<0.05$ HFD + saline vs. HFD + NTG. TNF, tumor necrosis factor; NTG, nitroglycerin; HFD, high fructose diet; SEM, standard error of the mean. 
Table 2 Biochemical metabolic parameters in water control group (water + saline), HFD control group rats (HFD + saline), NTG-injected rats (water + NTG), and HFD NTG-injected rats (HFD + NTG), with or without n-3 supplementation (water + n-3; HFD + n-3; n-3 + NTG; HFD $+\mathrm{n}-3+\mathrm{NTG})$

\begin{tabular}{|c|c|c|c|c|c|c|c|c|}
\hline $\begin{array}{l}\text { Biochemical } \\
\text { parameters }\end{array}$ & \multicolumn{4}{|c|}{ Water } & \multicolumn{4}{|c|}{ HFD } \\
\hline $\begin{array}{l}\text { Total cholesterol } \\
(\mathrm{mg} / \mathrm{dL})\end{array}$ & $82.0 \pm 8.0^{\mathrm{a}, \mathrm{b}, \mathrm{c}}$ & $55.7 \pm 8.5^{\mathrm{b}, \mathrm{e}}$ & $47.4 \pm 8.0^{c}$ & $38.0 \pm 7.0^{\mathrm{e}}$ & $\begin{array}{l}63.0 \pm 3.6^{a} \\
(P=0.054)\end{array}$ & $61.0 \pm 3.5^{i}$ & $\begin{array}{l}46.0 \pm 2.0 \\
(P=0.054)\end{array}$ & $43.5 \pm 3.4^{i}$ \\
\hline $\mathrm{HDL}(\mathrm{mg} / \mathrm{dL})$ & $59.0 \pm 5.2^{\mathrm{b}, \mathrm{c}}$ & $\begin{array}{l}32.4 \pm 8.0^{b} \\
(P=0.051)\end{array}$ & $38.6 \pm 2.4^{c, d}$ & $\begin{array}{l}20.0 \pm 4.5^{d} \\
(P=0.051)\end{array}$ & $47.0 \pm 5.6$ & $39.0 \pm 1.4^{i}$ & $35.0 \pm 2.0$ & $26.0 \pm 1.5^{i}$ \\
\hline
\end{tabular}

Data are presented as means \pm SEM of 10 to 12 rats per group for glycemia, 5 to 6 rats per group for triglycerides, total cholesterol, HDL and non-HDL cholesterol (one-way ANOVA followed by uncorrected Fisher's LSD's post hoc; or Kruskal-Wallis followed by uncorrected Dunn's post hoc test). ${ }^{a}, \mathrm{P}<0.05$ water + saline vs. HFD + saline; ${ }^{b}, \mathrm{P}<0.05$ water + saline vs. water $+\mathrm{NTG} ;{ }^{c}, \mathrm{P}<0.05$ water + saline vs. water + n-3; ${ }^{d}, \mathrm{P}<0.05$ water + n-3 vs. water + n-3 + NTG; ${ }^{e}$, water + NTG vs. water + n-3 + NTG; ${ }^{\circ}, \mathrm{P}<0.05$ HFD + saline vs. HFD + NTG; ', P<0.05 HFD + NTG vs. HFD + n-3 + NTG; P=0.09 for water + n-3 vs. water + n-3 + NTG, $\mathrm{P}=0.08$ for water + n-3 + NTG vs. HFD + n-3 + NTG. NTG, nitroglycerin; HFD, high fructose diet; SEM, standard error of the mean.

NTG-injected rats and tap water control rats. There was a slight decrease $(\mathrm{P}=0.09)$ in glycemia levels with NTG injections in $n-3$ treated tap water rats, compared with $n-3$ treated NTG-free tap water rats. Interestingly, n-3 treated HFD NTG-injected rats presented a modest increase $(\mathrm{P}=0.08)$ in glycemia levels than tap water rats with the same treatments. Concerning triglycerides, we observed a trend to decrease of this parameter in HFD NTG-injected rats, compared with their NTG-free counterparts $(\mathrm{P}=0.07)$; no changes were registered for NTG or n-3 treatments. HFD rats showed decreased total cholesterol in comparison with tap water rats. Also, a reduction of total cholesterol was observed in tap water NTG-injected rats, in relation to tap water NTG-free rats. N-3 treatment decreased the total cholesterol of tap water rats, while HFD rats displayed a slight decrease of total cholesterol by $n-3$ treatment $(\mathrm{P}=0.054)$. Total cholesterol was decreased in $\mathrm{n}-3$ treated rats, either in the tap water NTG-injected group or the HFD NTG-injected group, compared with their n-3-free counterparts.

Concerning the HDL cholesterol fraction, tap water NTG-injected rats displayed decreased HDL cholesterol than the NTG-free tap water rats. Unexpectedly, we observed a decrease in this parameter in the n-3 treated tap water rats compared with the $\mathrm{n}-3$-free control group. Also, there was a slight decrease in HDL cholesterol in the $\mathrm{n}-3$ treated tap water NTG-injected rats, compared with the $\mathrm{n}-3$-free tap water NTG-injected rats $(\mathrm{P}=0.051)$. $\mathrm{N}-3$ treatment was able to decrease the HDL cholesterol in HFD NTG-injected rats compared with their n-3-free counterparts. We did not observe any differences for the non-HDL cholesterol among the experimental groups (Table 2).

\section{Assessment of affective behavior}

We looked over to the anxiety behavior by using the EPM, $24 \mathrm{~h}$ after each NTG injection (Table 3). The HFD group had a significant increase in the total number of entries in the EPM, compared with the tap water control group. Furthermore, there was a decrease in the total number of entries for rats that received NTG injections, either in the HFD or in the tap water group. The $\mathrm{n}-3$ treatment had no effect on this parameter. Also, the NTG injections significantly reduced the number of entries in open arms, regardless of HFD supplementation, compared with their 
Table 3 Elevated plus maze (EPM) behavior assessed $24 \mathrm{~h}$ after NTG injections in: water control group rats (water + saline), HFD control group rats (HFD + saline), NTG-injected rats (water + NTG), HFD NTG-injected rats (HFD + NTG), rats supplemented with n-3 (water + n-3), HFD rats supplemented with n-3 (HFD + n-3), NTG-injected rats supplemented with n-3 (n-3 + NTG) and HFD NTG-injected rats supplemented with n-3 (HFD + n-3 + NTG)

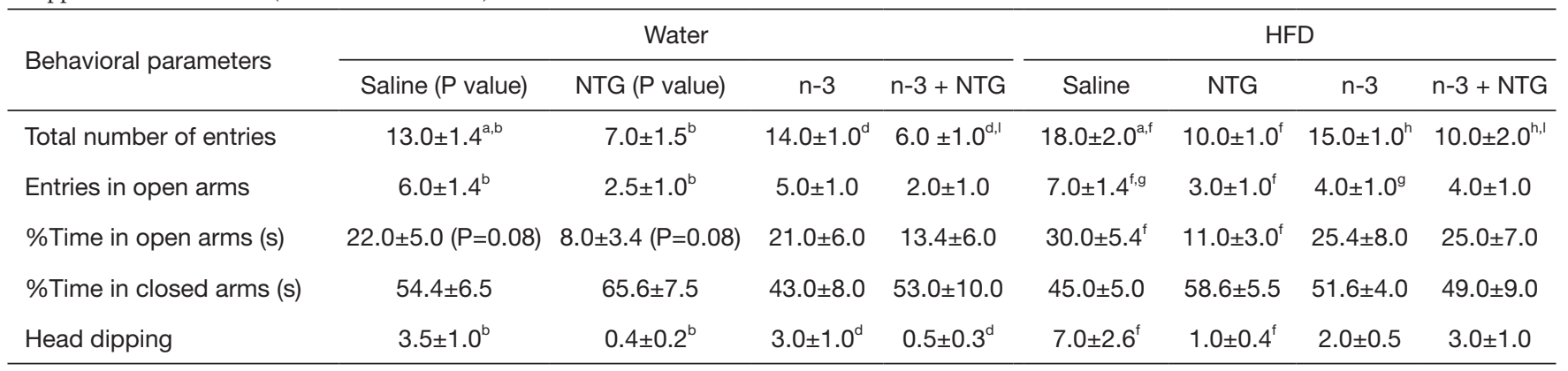

Data are presented as means \pm SEM of 10 to 12 rats per group (one-way ANOVA followed by uncorrected Fisher's LSD's post hoc; or Kruskal-Wallis followed by uncorrected Dunn's post hoc test). ${ }^{a}, \mathrm{P}<0.05$ water + saline vs. HFD + saline; ${ }^{b}, \mathrm{P}<0.05$ water + saline vs. water + NTG; ${ }^{d}, \mathrm{P}<0.05$ water + n-3 vs. water + n-3 + NTG; ${ }^{\mathrm{f}}, \mathrm{P}<0.05 \mathrm{HFD}+$ saline vs. HFD + NTG; ${ }^{9}, \mathrm{P}<0.05 \mathrm{HFD}+\mathrm{saline}$ vs. HFD + n-3; ${ }^{\mathrm{h}}$, $\mathrm{P}<0.05$ HFD + n-3 vs. HFD + n-3 + NT; ', P<0.05 water + n-3 + NTG vs. HFD + n-3 + NTG. NTG, nitroglycerin; HFD, high fructose diet; SEM, standard error of the mean.

NTG-free counterparts. N-3 treatment decreased the number of entries in open arms only in the HFD group.

As for the percentage of time spent in open arms, we observed a slight decrease of this parameter in the tap water NTG-injected rats compared with tap water control group $(\mathrm{P}=0.08)$. There was a significant decrease of the percentage of the time spent in the open arms for the HFD NTG-injected rats, when compared with their NTG-free counterparts. The n-3 treated HFD NTG-injected rats did not display any changes in the percentage of time spent in open arms, compared with n-3 treated HFD NTGfree rats, despite the lack of significant differences when comparing HFD NTG-injected rats, with and without n-3. This result indicates that NTG injections did not exert affective alterations in rats that had been treated with $n-3$. As for the percentage of time spent in the closed arms, there were no significant differences among the groups.

About the number of head dippings, we noted a decrease of head dippings in the NTG-injected rats, both in the tap water rats or in the HFD rats, when compared with their control mates. N-3 treated HFD rats that received NTG injections did not display reduced exploratory behavior as the n-3-free HFD rats with NTG injections (Table 3).

\section{Discussion}

The present study investigated the impacts of HFDinduced MS in a rat model of migraine evoked by NTG administration, aiming to analyze the effects of n-3 supplementation in this scenery. To achieve this goal, we looked over the influence of HFD and n-3 supplementation on pain-like intensity, photophobia, adiposity, inflammatory markers, adipokines, biochemical profiling, and the affective behavior endpoints related to the induction of migraine-like changes by repeated NTG injections.

Initially, we hypothesized that MS generated by HFD could exacerbate the migraine-like symptoms. This premise was based on previous studies suggesting that migraine could be aggravated in obese children and adults $(7,8,18-20)$. Surprisingly, our data revealed that HFD alleviated the pain-like behavior caused by NTG injections. In the present study, five NTG injections led to photophobia and pronounced migraine-like pain behavior, as assessed by the dark/light box and grimace scores, respectively, in accordance with previous studies $(14,21)$. The NTG injections also diminished food intake, either in the tap water or in the HFD group, a feature that was described elsewhere (22). Moreover, we observed an important reduction of whole body and epididymal fat weights and a mild reduction of inguinal fat weight in the tap water NTGinjected rats. The decrease of food intake and body weight by NTG injections might reflect the reduced appetite and the presence of nausea, which are common migraine symptoms (22). Otherwise, NTG-injected rats consuming HFD showed body weight gain, despite a mild reduction of inguinal adipocyte area and size frequency, epididymal fat weight and the respective adipocyte size frequency. Also, HFD NTG-injected rats displayed reduced glycemia when 
compared with the HFD control rats. The increase in body weight might suggest a protective role for fructose ingestion in migraine, correlating with the diminished grimace scale scores in this group. One has reported that ghrelin can relief pain, restoring body weight and appetite in rats submitted to NTG injections (22). Furthermore, it was previously observed that maternal fructose supplementation can increase plasma ghrelin levels in dams and pups (23), which is supposed to relieve the painful responses by activating the mesolimbic dopaminergic circuit, leading to reward and motivation-related feelings (22). Another mechanism that could support our findings is that fructose can decrease the cerebral blood flow, evoking feeding behavior in rodents (24). Also, there is evidence suggesting that fructose can reduce the eNOS activation, consequently preventing the nitric oxide (NO) production (25). In fact, NO is able to increase the cerebral blood flow, being the vasodilator agent released by NTG (25-27).

The disfunction in sensory processing in migraine might lead the brain to demand greater amounts of energy (28). It has been suggested that a migraineur brain has decreased organic phosphates because of fallen oxidative phosphorylation (29-31) and a glucose hypometabolism in brain regions linked with nociceptive processing (32). Some authors suggest that the combination of greater energy expenditure with reduced energy storage could activate the trigeminovascular system, triggering migraine (33). There is evidence that the degree of insulin resistance (IR) positively correlated with chronic migraine (34), which could be an adaptive counter regulatory response that impairs the entry of glucose into peripheral tissues, thereby increasing the glucose availability in the brain (33). This mechanism explains why T2DM might be protective against migraine $(35,36)$. Herein, we observed that NTG injections reduced the blood glucose levels in HFD rats, which could imply an increase of insulin sensitivity in this group.

Some authors have suggested that $\mathrm{n}-3$ supplementation along with amitriptyline might help to decrease migraine frequency (37). Herein, there were no changes of photophobia or pain scores after one month of n-3 supplementation. A reduction of headache duration and severity, besides life quality, has been demonstrated in patients supplemented with n-3 (38). Nonetheless, a metaanalysis did not reveal any correlation between $n-3$ intake and migraine severity or frequency, despite a reduction of migraine duration per day (39). Further studies with longer $\mathrm{n}-3$ treatment protocols and different doses are required for more conclusive results.
We observed a decrease of epididymal adipocyte weight in the NTG-injected rats, both in the HFD or in the tap water rats compared with their NTG-free control rats, which could suggest an increased lipolysis in this tissue, as the visceral WAT presents a higher lipolytic activity due to the greater presence of $\beta 3$-adrenergic receptors (40). Some authors have observed increased cortisol levels in migraineurs (41-43), as well as an elevation of plasma free fatty acids and glycerol (44), which might suggest a protective response via adipose tissue lipolysis for compensating the high energy demand (33). Metabolic alterations during energy deprivation include suppression of anabolic pathways and stimulation of catabolic pathways, such as the glucose uptake and fatty acid oxidation, two processes that act together to restore ATP levels (45). The increase in inguinal fat weight in the HFD NTG-injected rats compared with the tap water NTG-injected rats might support a defensive role for fructose in migraine.

In this study, the $\mathrm{n}-3$ treatment did not exert any significant effect on body weight change in the tap water NTG-injected rats. Conversely, in the HFD NTG-injected rats, n-3 treatment prevented the body weight increase in this group. This effect might be due to the increased energy expenditure with n-3 supplementation (46), combined with the decreased food intake observed in the NTG-treated rats. The effect of $n-3$ might not be evident in the tap water NTG-injected rats because their body weight was lesser than the body weight of HFD NTG-injected rats.

$\mathrm{N}-3$ along with NTG injections potentiated the decrease in inguinal adipocyte size in the tap water rats. It is well documented that $n-3$ can induce browning in inguinal (47) and epididymal (48) adipocyte tissues, thus increasing the thermogenesis (47). Until now, there is no evidence regarding $\mathrm{n}-3$ effects on the adipose tissue of NTG-injected rats. Nonetheless, the effects of $n-3$ on inguinal adipocyte size in NTG-injected rats might be due to NTG-induced decrease in food intake (22), combined with increased n-3induced thermogenesis in inguinal adipose tissue (47). Furthermore, there is evidence that $\mathrm{n}$-3-induced thermogenesis might be dependent on TRPV1 activation (46), and it is known that migraine-like pain induced by NTG injections is mediated by this same receptor (22). There might be an overstimulation of TRPV1 channels leading to enhanced thermogenesis in the n-3 treated tap water NTG-injected rats. As expected, n-3 supplementation was able to decrease epididymal adipocyte size in the tap water rats and in the HFD rats, the last one evidenced by the adipocyte area frequency size. Similar results were 
seen elsewhere, whose authors suggested that $n-3$ can increase epididymal fat utilization through mitochondrial uncoupling and peroxisomal fatty acid oxidation (48). Strikingly, n-3 treatment was unable to decrease epididymal fat weight and size in the HFD NTG-injected rats. It seems that $\mathrm{n}-3$ preserved the epididymal fat somehow, despite the decreased body weight of n-3 treated HFD NTGinjected rats compared with their n-3-free counterparts. Some studies reported increased visceral adipose tissue mass with n-3 treatment, and this has been associated with an amelioration of inflammation and metabolic parameters (49).

The CLS is a marker of macrophage infiltration in the adipose tissue, which can lead to cell death through pyroptosis (50). Remarkably, it has been previously demonstrated that pyroptosis mostly occurs in hypertrophic adipocytes (51). In our study, the HFD group presented higher CLS intensity than tap water rats, according to the evaluation of inguinal subcutaneous adipose tissue, which is likely related to an increase of HFD inguinal adipocytes. On the other hand, some authors suggested that adipocyte death is more prevalent in epididymal adipose tissue of mice (52) and rats (53) than in subcutaneous adipose tissue. However, individuals with MS showed increased macrophage infiltration and CLS in subcutaneous adipose tissue, and the amount of subcutaneous adipose tissue in these individuals was positively correlated with MS and IR degree (54). We observed an increase of CLS intensity in both inguinal and epididymal fat in the tap water NTGinjected rats compared with the NTG-free tap water rats. The increase in CLS might point out to a pro-inflammatory state, with decreased insulin sensitivity (50). A previous study demonstrated that exercise training associatedlipolysis led to increased inflammation in the subcutaneous adipose tissue, providing lipids for muscle energy, allowing to suggest that, in our study, the increased CLS numbers in inguinal fat - a pro-inflammatory process — of tap water NTG injected rats could be an indicative of lipolysis (55). Indeed, the increase in the epididymal CLS intensity in the HFD NTG-injected rats suggests a higher lipolysis in this group. The inflammation of the adipose tissue could be a hint to fatty acid oxidation (55), corroborating with the decreased epididymal fat depot weight in this group. The CLS was absent in the inguinal adipose tissue of HFD NTG-injected rats, possibly indicating an improvement of IR in the HFD NTG-injected rats compared with their NTG-free control rats. Hence, Jialal et al. suggested that a reduction of adipose tissue inflammation in MS might suggest an improved insulin sensitivity (54); however, this study was performed in the subcutaneous adipose tissue and there is no evidence of this process in the visceral adipose tissue. Another indication of HFD protection in migraine is the higher inguinal CLS intensity in the tap water NTG-injected rats than in the HFD NTG-injected rats, indicating decreased IR in the last group.

Herein, we observed an increased CLS intensity in the visceral fat depot of the n-3 treated HFD NTG-injected group. In conditions like fasting, the visceral adipose tissue might be protective via oxidation reaction, as this fat can better drain to the portal vein circulation compared with the subcutaneous fat, being more efficient as for energy demands, preserving energy homeostasis (56). Some authors suggest that migraine occurs in a sparse glucose condition, similar to fasting (33). Taking in account that n-3 treatment can increase energy expenditure (46), and increased visceral fat weight was seen in fasted rats previously supplemented with fructose (57), this effect might occur in our experimental paradigm. Also, increased visceral CLS intensity in this group might arise due to hypertrophic adipocytes (52), as n-3 treatment was unable to decrease adipocyte size in this group, despite the body weight decrease. There was a greater decrease in the CLS intensity in the subcutaneous fat of n-3-treated HFD rats, when compared with their $\mathrm{n}$-3-free counterparts. Spencer et al. observed that n-3 decreased the pro-inflammatory macrophages and subcutaneous CLS in patients with MS (58). Considering that adipocyte enlargement is one of the CLS-inducing factors (59), we detected a mild decrease in the adipocyte areas of $n-3$ treated HFD rats. Therefore, decreased CLS intensity might occur due to n-3-induced improvement of adipocyte hypoxia and necrosis. N-3 treatment significantly decreased the CLS intensity in subcutaneous and in visceral fat of tap water NTG-injected rats. Until now, there is no evidence of $n-3$ effects in adipose tissue of NTG-injected rodents. Nonetheless, it has been suggested that infiltrated macrophages in adipose tissue can contribute to systemic inflammation and IR (60). Thus, it is possible that n-3 might reduce adipose tissue inflammation, as it diminishes the systemic inflammation as well (58).

CGRP is a potent cerebral vasodilator, released by trigeminal nerves, being considered a neuromodulator of the central pain circuitries $(61,62)$. Previous reports showed that NTG is capable of increasing plasma CGRP levels (14). However, we did not observe an increase in the serum CGRP levels, neither in the HFD nor in the tap water NTG-injected rats. Lee et al. did not find any correlation 
between serum CGRP levels and migraine episodes in chronic migraineur patients (63). Nevertheless, there was a slight decrease of CGRP in the HFD NTG-injected rats, compared with the tap water NTG-injected rats. Fructose can lead to a serum CGRP decrease and enhanced production of vasoconstrictor mediators in rats (64), which could help to counteract NTG effects on development of migraine-like alterations. Noteworthy, there was a decrease in serum CGRP with n-3 treatment in the tap water NTGinjected rats. A recent evidence showed that the n-3-derived resolvin D3 was able to inhibit the TRPV1 currents and the release of CGRP in psoriatic mice (65), supporting somewhat our results. Conversely, serum CGRP was not altered in the n-3 treated HFD NTG-injected rats, maybe because serum CGRP levels of n-3-free HFD NTGinjected rats were already low.

The hypothalamic CGRP levels were decreased in the HFD rats, when compared with the tap water rats. Substantiating our results, previous evidence showed an increased signaling of glucocorticoid receptor and ghrelin levels during fructose metabolism $(23,66)$, which might be responsible for the hypothalamic CGRP decline under HFD (67). Surprisingly, hypothalamic CGRP was increased in the HFD NTG-injected rats compared with their NTG-free counterparts. CGRP elevation can lead to fat accumulation in rodent models of obesity $(68,69)$. According to that, in the present study, there was an increase of fat accumulation in the HFD NTG-injected group compared with their NTG-free counterparts. The increased hypothalamic CGRP levels could be a compensatory response for reduce the energy expenditure with the purpose to decrease migraine energy needs, as mice lacking CGRP gene have an increased energy expenditure (69). Hypothalamic CGRP was not affected by n-3 treatment in any group. It has been hypothesized that migraine can be alleviated by $n-3$-enriched diet, through decreased inflammatory mediators (70). However, until now, there is no evidence for n-3 effects on hypothalamic CGRP of NTG-injected and MS rats, indicating the need of more research that evaluate the n-3 effect under different doses and periods when these conditions co-occur.

Herein, we observed diminished serum leptin levels in NTG-injected rats, in both HFD and tap water groups. A previous study has shown that leptin could alter trigeminal nerve nociceptive inputs to higher brain levels. The authors demonstrated that trigeminocervical neuronal firing was significantly inhibited by leptin infusion in rats (71), indicating a potentially antinociceptive effect for this adipokine. These results are consistent with the fact that fasting induces a decrease in plasma leptin levels and it is able to trigger migraine attacks (71). Clinical studies also indicate a disruption of GLP-2 and leptin levels in migraineurs $(72,73)$. Nonetheless, leptin levels are positively correlated with body fat mass (74) and, in our study, tap water NTG-injected rats displayed a reduction of this feature, when compared with NTG-free control rats. Thus, low leptin levels in NTG-injected rats could be a result of decreased fat mass. Still, the HFD NTG-injected rats had higher body fat mass than their NTG-free counterparts and still showed less leptin levels when compared with their NTG-free control group. There is evidence that a drop in leptin levels is accompanied by metabolic adaptations that limit the energy expenditure (75), which could be an adaptive advantage for preventing migraine energy needs. $\mathrm{N}-3$ treatment was able to decrease serum leptin levels in the tap water rats. Some authors suggested that $n-3$ lowering effects on leptin might occur only when combined with an energy-restricted diets (76). This hypothesis is in accordance with our results showing the absence of any serum leptin variation levels in the n-3-treated HFD rats.

Leptin acts on hypothalamic neurons to regulate food intake, blood glucose levels and thermogenesis (77). We did not observe significant hypothalamic leptin changes among the groups. Despite being able to capture leptin from the blood, some studies suggest that hypothalamus itself produces leptin (78). Until now, there is no study that evaluated hypothalamic leptin levels under migraine and/or MS conditions. One has shown that hypothalamic leptin levels in fasting rats was significantly changed only when the lipid stores were compromised. For instance, hypothalamic leptin levels only decrease after 12-h of fasting, different of serum leptin levels that vary after 6-h of fasting. The authors suggested that adipose leptin production has a role in the short-term food intake, while hypothalamic leptin is responsible for the long-term energy homeostasis regulation (79). N-3 treatment slightly reduced the hypothalamic leptin of HFD NTG-injected rats. Leptin act on hypothalamus triggering an increase in energy expenditure through its effects on cardiovascular system and adipose tissue thermogenesis (80). Ahlma et al. showed that starvation leads to decreased hypothalamic leptin levels, accompanied by metabolic adaptation that limits energy expenditure (75). Thus, as migraine might require more brain fuel, and n-3 leads to an increase in energy expenditure, a mild decrease of hypothalamic leptin in the n-3-treated HFD NTG-injected rats might be a 
compensatory response to maintain energy homeostasis.

It is believed that adiponectin has a protective role against IR and atherosclerosis development, and also displays anti-inflammatory properties, regulating lipid and glucose metabolism (81). Plasma adiponectin is divided in low-, medium-, and high-molecular weight forms, which activate different pathways and have distinct physiological functions (82). The high molecular weight adiponectin seems to have pro-inflammatory effects, whereas the low molecular weight adiponectin might have anti-inflammatory properties (83). Herein, we measured the total adiponectin fraction. There is a consensus that decreased levels of total adiponectin is associated with IR, T2DM, obesity, and MS (81). Because of the pro-inflammatory effects of decreased adiponectin levels, literature data suggests that this mediator plays a relevant role in migraine (84). In the present study, HFD rats displayed decreased total serum adiponectin levels, when compared with the tap water group, suggesting a probable increase of IR in the HFD group. However, this difference was absent in the NTG-injected rats. It is worth mentioning that we cannot exclude that there were no differences in the proportion of the different molecular adiponectin fractions in our study. Unexpectedly, serum adiponectin levels were decreased in all n-3 treated groups. Ramos-Romero et al. reported no significant changes in serum adiponectin levels in the n-3 treated rats (85), while some authors documented increased (86) or decreased adiponectin levels (87), the last one corroborating with the present data, although it was associated with omega-6 (n-6) supplementation. The leptin:adiponectin ratio has been pointed out as a better indicator of inflammatory status (88). Unfortunately, we could not evaluate that because samples were insufficient to measure both leptin and adiponectin in samples obtained from the same animal. Despite, recent studies have shown that adiponectin might have a dual role, either anti- or pro-inflammatory, according to its isoform and the target tissue (89). This suggests the need for further investigation about the n-3 effects on serum adiponectin isoforms in the migraine and MS framework.

Adiponectin receptors AdipoR1 and AdipoR2 are expressed in the CNS, and specifically in the hypothalamus, with the AdipoR1 playing a role in insulin sensitivity regulation (90). There is an inverse correlation between adiponectin levels and IR (91,92). Furthermore, a series of studies in rodents has demonstrated that in the CNS, adiponectin inhibits food intake and stimulates energy expenditure, leading to decreased body weight $(93,94)$. Also, adiponectin enhances insulin action leading to suppression of liver glucose production-i.e., gluconeogenesis-, and thus, it lowers the plasma glucose and fatty acid levels, stimulating fatty acid oxidation by skeletal muscle (95). In our study, there was a decrease of hypothalamic adiponectin levels in the HFD NTG-injected rats, compared with the HFD control rats, a feature that was also seen in the $n-3$ treated tap water rats injected with NTG, compared with n-3 treated NTG-free control rats. NTG might reduce hypothalamic adiponectin, so that the mechanisms above are suppressed, helping to let available circulating glucose and fatty acids for usage by the brain in an energy crisis, as it occurs in migraine (33). As adiponectin in the CNS inhibits food intake, stimulates energy expenditure $(93,94)$ and enhances insulin action $(91,92)$, the reduction of hypothalamic adiponectin might be a protective response to increased energy demands in NTG-injected rats, worsened by higher energy expenditure under n-3 treatment.

HFD rats displayed a slight decrease of hypothalamic IL-6 levels when compared with the tap water rats. Previous studies have shown a decreased IL-6 expression in the parabrachial nucleus of obese male and female mice (96). The actions of IL-6 in the periphery and in the central nervous system can differ; for instance, increased IL-6 serum levels in obesity did not reflect increased brain IL-6 levels. On the contrary, obese and overweight men displayed decreased IL-6 levels in the cerebrospinal fluid, being negatively correlated with body fat mass (97). Also, there was a modest decrease of hypothalamic IL-6 in the HFD NTG-injected rats compared with the tap water NTG-injected rats. As the overexpression of central IL-6 can increase energy expenditure and decrease body weight $(98,99)$, this reduction of hypothalamic IL-6 might suggest a drop of energy expenditure in the HFD NTG-injected rats, fixing the migraine energy needs. We observed decreased hypothalamic IL-6 levels only in the tap water rats treated with n-3. Conversely, others have observed an inverse relationship between n-3 and IL-6 levels in humans (100). More studies with different n-3 doses and longer treatment duration are required to confirm n-3 effects on neuroinflammation.

High hypothalamic TNF contents reflects an inflammatory damage, which is one of the mechanisms involved in the obesity pathophysiology (101). Some authors suggest that the inflammation and hypothalamic dysfunction present in obesity and in migraine can contribute to the development of each other. Also, they suggest that the prescription of low glycemic diet could 
contribute to migraine improvement by decreasing neuroinflammation (70). Nonetheless, we did not observe significant hypothalamic TNF alterations in neither the HFD nor in the tap water NTG-injected rats. Opposing to our result, an increased mRNA expression of hypothalamic TNF has been shown after 8 weeks of high fat diet (102). Additionally, an elevation of hypothalamic TNF levels was seen following 56 days of $30 \%$ fructose supplementation in rats (103). There is evidence that after some days of neuroinflammation, a neuroprotective response occurs, triggering a decrease of inflammatory markers, with a recovery of enhanced levels within 4 weeks of high fat feeding (104), reflecting hypothalamic TNF levels oscillations. One study has reported only one peak of TNF mRNA expression, after $4 \mathrm{~h}$ of cortical spreading depression, which was resolved by $12 \mathrm{~h}$ (105). Hypothalamic $\mathrm{TNF} \alpha$ levels significantly decreased with $\mathrm{n}-3$ treatment in the tap water NTG-injected rats. It has been demonstrated that endogenous $n-3$ is related to decreased hypothalamic TNF levels in mice (106). Besides, $n-3$ supplementation led to a reduction of TNF $\alpha$ gene expression in NTG-injected patients (107). However, there is no evidence of reduced hypothalamic TNF $\alpha$ levels in NTG-injected rats, until now. There is a neuroinflammatory component in migraine pathogenesis and the n-3 treatment might attenuate this inflammation by decreasing TNF $\alpha$ levels (107).

Symptoms of anxiety and depression are frequent in individuals with MS (108) and migraine (109). Although not completely solved, the underlying mechanisms of MS are likely related to a low grade chronic inflammation, leading to impaired biochemical and hypothalamic pathways (108). Migraine-related affective behaviors have been associated with hypothalamic dysfunction and inflammation (110), as well. That said, IR has been described during major depression, suggesting impaired glucose utilization, similar to T2DM (111). Herein, HFD rats presented hyperactivity, as indicated by the greater number of entries in arms in the EPM. It has been documented that fructose intake can lead to hyperactive foraging response that stimulates impulsivity and increases the risk of developing deficit hyperactivity disorder (112). This effect might be related to increased uric acid driving to greater locomotion index, impulsivity and exploratory activity, as it was documented in rats (113) and humans (114). Nevertheless, chronic HFD can drive to desensitization of hedonic responses and to depression (112). Alternatively, at the end of migraine-induction protocol, NTG injections were able to decrease the total number of entries and head dipping bouts, either in the HFD rats or in the tap water rats. Furthermore, NTG injections decreased the entries in the open arms and the percentage of time spent in open arms, indicating minor locomotor activity and an anxiogenic behavior in NTG-injected rats. Similar results were described before in rats that had been treated with NTG, which is likely driven by increased cortisol levels (115).

There are substantial evidence that $\mathrm{n}-3$ can relief symptoms of affective-emotional disorders $(100,116)$. In fact, n-3 was able to partly decrease the anxiety-like behavior in NTG-injected rats, as evidenced by the lack of significant difference in the percentage of time spent in open arms between HFD n-3 treated NTG-free rats and their n-3 treated NTG-injected counterparts, while there was a significant difference between HFD n-3- free control rats and HFD n-3-free NTG-injected rats. Importantly, this is the first data demonstrating the effects of $\mathrm{n}-3$ treatment in affective-behavior related to migraine in rats. Nonetheless, prior evidence showed antidepressant effects for $n-3$ in a stress model in rats (117), corroborating with the present data. Also, n-3 was able to mitigate the fructose-induced hyperactivity in rats, as indicated by decreased entries in open arms of n-3 treated HFD rats, when compared with n-3 free HFD animals. Additionally, one has shown a significant improvement of clinical symptoms in children and adolescents with attention deficit hyperactivity disorder after supplementation with n-3 (118), rather supporting our results.

Our study has some limitations that require to be discussed. Concerning the NTG model, it has been described that it can reproduce the main components of migraine in rodents and humans $(119,120)$. However, it is relevant to mention that the model has some restrictions, such as a different pattern of responses depending on the rodent species and sex, or the previous history of migraine in humans $(12,119)$. As for the inflammatory mediators' analysis, whereas isoflurane does not seem to alter the CGRP levels (121), it can affect the brain contents of proinflammatory cytokines, as described beforehand (122). However, the differences among the groups can be considered consistent, as all of the animals were submitted to euthanasia by isoflurane inhalation, under the same protocol.

\section{Conclusions}

The present study highlights the role of fructose-related metabolic pathways in migraine pathogenesis and the impact of n-3 treatment in this context. Remarkably, the 


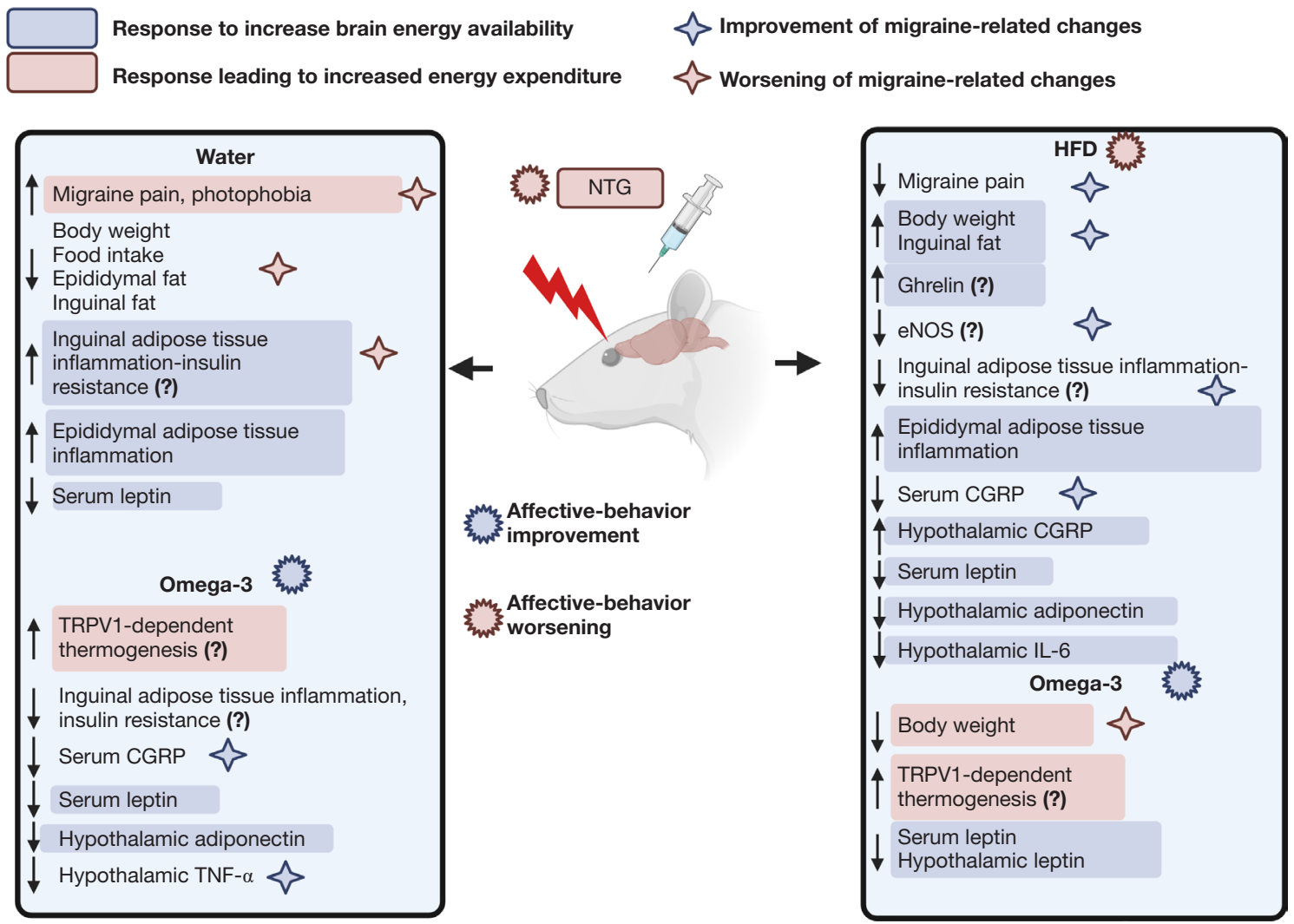

Figure 9 Overview of the main findings of the present study. The blue rectangles indicate the situations in which there is a response to increase brain energy availability. Alternatively, the pink rectangles are indicative of responses triggering increased energy expenditure. Blue and pink stars show improvement and worsening of migraine-like changes, respectively. The blue and pink spiked circles show improvement and worsening of affective-behavior changes, correspondingly. The question marks represent the issues that need further investigation. Figure created with BioRender.com. CGRP, calcitonin gene-related peptide; e-NOS, endothelial nitric oxide synthase; IL-6, interleukin-6; HFD, high-fructose diet; NTG, nitroglycerin; TNF- $\alpha$, tumor necrosis factor- $\alpha$; TRPV-1, transient receptor potential vanilloid 1.

HFD relieved migraine-related pain, although it did not affect photophobia. HFD also led to an enhancement of some biochemical and anthropometric parameters related to the NTG-induced migraine in rats. One month of $n-3$ treatment was unable to ameliorate migraine pain, besides it impaired some of the favorable behavioral and biochemical effects of HFD on NTG-injected rats. However, there was a decrease of some inflammatory markers in the n-3 treated tap water NTG-injected rats, as well as a modulation of affective parameters. The improvement of NTG-related changes by HFD could be due to responses leading to an increase of energy availability, as the migraineur brain might requires more amounts of energy. In line with this, the prevention of HFD-related changes by $n-3$ treatment could be associated with an increased energy expenditure. A summary of these propositions is outlined in Figure 9.
Future research should be conducted to investigate mitochondrial metabolism and ATP production in the MS and migraine framework. Also, there is a need to look over the effects of $n-3$ supplementation in long-term schedules of treatment, as well as the effects of other obesity-induced diets on migraine. Nonetheless, our data helps to pave the way regarding the relationship between MS and migraine, besides the effects of $n-3$ supplementation in this context.

\section{Acknowledgments}

We would like to thank Mrs. Janaína Pasetti Nunes and M.Sc. Gabriel Rubensam for their excellent technical assistance and Dr. Jarbas Rodrigues de Oliveira for his generous support with the biochemical kits (Labtest, Brazil). MMC is a researcher career awardee of the Conselho 
Nacional de Desenvolvimento Científico e Tecnológico (304042/2018-8).

Funding: This work was supported by grants from the Coordenação de Aperfeiçoamento de Nível Superior (CAPES; Financial Code 001), Conselho Nacional de Desenvolvimento Científico e Tecnológico (CNPQ) and Fundação de Amparo à Pesquisa do Estado do Rio Grande do Sul (FAPERGS).

\section{Footnote}

Reporting Checklist: The authors have completed the ARRIVE reporting checklist. Available at https://atm. amegroups.com/article/view/10.21037/atm-21-5699/rc

Data Sharing Statement: Available at https://atm.amegroups. com/article/view/10.21037/atm-21-5699/dss

Conflicts of Interest: All authors have completed the ICMJE uniform disclosure form (available at https://atm. amegroups.com/article/view/10.21037/atm-21-5699/coif). The authors have no conflicts of interest to declare.

Ethical Statement: The authors are accountable for all aspects of the work in ensuring that questions related to the accuracy or integrity of any part of the work are appropriately investigated and resolved. Experiments were performed under a project license (9088) granted by Institutional Animal Care and Use Committee of PUCRS on December 5, 2018, in compliance with the current Brazilian guidelines for the care and use of animals.

Open Access Statement: This is an Open Access article distributed in accordance with the Creative Commons Attribution-NonCommercial-NoDerivs 4.0 International License (CC BY-NC-ND 4.0), which permits the noncommercial replication and distribution of the article with the strict proviso that no changes or edits are made and the original work is properly cited (including links to both the formal publication through the relevant DOI and the license). See: https://creativecommons.org/licenses/by-nc$\mathrm{nd} / 4.0 \%$.

\section{References}

1. Institute for Health Metrics and Evaluation (IHME). GBD Compare Data Visualization. 2018 [cited 2020 Jun 22]. Available online: http://vizhub.healthdata.org/gbd- compare

2. Kokavec A. Migraine: A disorder of metabolism? Med Hypotheses 2016;97:117-30.

3. Alberti KG, Eckel RH, Grundy SM, et al. Harmonizing the metabolic syndrome: a joint interim statement of the International Diabetes Federation Task Force on Epidemiology and Prevention; National Heart, Lung, and Blood Institute; American Heart Association; World Heart Federation; International Atherosclerosis Society; and International Association for the Study of Obesity. Circulation 2009;120:1640-5.

4. Serhan CN. Pro-resolving lipid mediators are leads for resolution physiology. Nature 2014;510:92-101.

5. Rajapakse T, Pringsheim T. Nutraceuticals in Migraine: A Summary of Existing Guidelines for Use. Headache 2016;56:808-16.

6. Becic T, Studenik C. Effects of Omega-3 Supplementation on Adipocytokines in Prediabetes and Type 2 Diabetes Mellitus: Systematic Review and Meta-Analysis of Randomized Controlled Trials. Diabetes Metab J 2018;42:101-16.

7. Bigal ME, Lipton RB, Holland PR, et al. Obesity, migraine, and chronic migraine: possible mechanisms of interaction. Neurology 2007;68:1851-61.

8. Bigal ME, Lipton RB. Obesity is a risk factor for transformed migraine but not chronic tension-type headache. Neurology 2006;67:252-7.

9. Streel S, Donneau AF, Dardenne N, et al. Screening for the metabolic syndrome in subjects with migraine. Cephalalgia 2017;37:1180-8.

10. Peterlin BL, Sacco S, Bernecker C, et al. Adipokines and Migraine: A Systematic Review. Headache 2016;56:622-44.

11. Kilkenny C, Browne W, Cuthill IC, et al. Animal research: reporting in vivo experiments--the ARRIVE guidelines. J Cereb Blood Flow Metab 2011;31:991-3.

12. Tavares CO, Rost FL, Silva RBM, et al. Cross Talk between Apical Periodontitis and Metabolic Disorders: Experimental Evidence on the Role of Intestinal Adipokines and Akkermansia muciniphila. J Endod 2019;45:174-80.

13. Caudle RM, Caudle SL, Flenor ND, et al. Pharmacological Characterization of Orofacial Nociception in Female Rats Following Nitroglycerin Administration. Front Pharmacol 2020;11:527495.

14. Mahmoudi J, Mohaddes G, Erfani M, et al. Cerebrolysin attenuates hyperalgesia, photophobia, and neuroinflammation in a nitroglycerin-induced migraine 
model in rats. Brain Res Bull 2018;140:197-204.

15. Al-Rasheed N, Al-Rasheed N, Bassiouni Y, et al. Potential protective effects of Nigella sativa and Allium sativum against fructose-induced metabolic syndrome in rats. J Oleo Sci 2014;63:839-48.

16. Lucena CF, Roma LP, Graciano MF, et al. Omega-3 supplementation improves pancreatic islet redox status: in vivo and in vitro studies. Pancreas 2015;44:287-95.

17. Fischer AH, Jacobson KA, Rose J, et al. Hematoxylin and eosin staining of tissue and cell sections. CSH Protoc 2008;2008:pdb.prot4986.

18. Eidlitz Markus T, Toldo I. Obesity and Migraine in Childhood. Curr Pain Headache Rep 2018;22:42.

19. Hershey AD, Powers SW, Nelson TD, et al. Obesity in the pediatric headache population: a multicenter study. Headache 2009;49:170-7.

20. Horev A, Wirguin I, Lantsberg L, et al. A high incidence of migraine with aura among morbidly obese women. Headache 2005;45:936-8.

21. Sufka KJ, Staszko SM, Johnson AP, et al. Clinically relevant behavioral endpoints in a recurrent nitroglycerin migraine model in rats. J Headache Pain 2016;17:40.

22. Farajdokht F, Mohaddes G, Shanehbandi D, et al. Ghrelin attenuated hyperalgesia induced by chronic nitroglycerin: CGRP and TRPV1 as targets for migraine management. Cephalalgia 2018;38:1716-30.

23. Kisioglu B, Nergiz-Unal R. Potential effect of maternal dietary sucrose or fructose syrup on CD36, leptin, and ghrelin-mediated fetal programming of obesity. Nutr Neurosci 2020;23:210-20.

24. Page KA, Chan O, Arora J, et al. Effects of fructose vs glucose on regional cerebral blood flow in brain regions involved with appetite and reward pathways. JAMA 2013;309:63-70.

25. Zhao CX, Xu X, Cui Y, et al. Increased endothelial nitricoxide synthase expression reduces hypertension and hyperinsulinemia in fructose-treated rats. J Pharmacol Exp Ther 2009;328:610-20.

26. Thomsen LL, Kruuse C, Iversen HK, et al. A nitric oxide donor (nitroglycerin) triggers genuine migraine attacks. Eur J Neurol 1994;1:73-80.

27. Frederiksen SD, Haanes KA, Warfvinge K, et al. Perivascular neurotransmitters: Regulation of cerebral blood flow and role in primary headaches. J Cereb Blood Flow Metab 2019;39:610-32.

28. Gantenbein AR, Sandor PS, Fritschy J, et al. Sensory information processing may be neuroenergetically more demanding in migraine patients. Neuroreport 2013;24:202-5.

29. Reyngoudt H, Paemeleire K, Descamps B, et al. 31P-MRS demonstrates a reduction in high-energy phosphates in the occipital lobe of migraine without aura patients. Cephalalgia 2011;31:1243-53.

30. Barbiroli B, Montagna P, Cortelli P, et al. Abnormal brain and muscle energy metabolism shown by $31 \mathrm{P}$ magnetic resonance spectroscopy in patients affected by migraine with aura. Neurology 1992;42:1209-14.

31. Lodi R, Montagna P, Soriani S, et al. Deficit of brain and skeletal muscle bioenergetics and low brain magnesium in juvenile migraine: an in vivo $31 \mathrm{P}$ magnetic resonance spectroscopy interictal study. Pediatr Res 1997;42:866-71.

32. Kim JH, Kim S, Suh SI, et al. Interictal metabolic changes in episodic migraine: a voxel-based FDG-PET study. Cephalalgia 2010;30:53-61.

33. Gross EC, Lisicki M, Fischer D, et al. The metabolic face of migraine - from pathophysiology to treatment. Nat Rev Neurol 2019;15:627-43.

34. Siva ZO, Uluduz D, Keskin FE, et al. Determinants of glucose metabolism and the role of NPY in the progression of insulin resistance in chronic migraine. Cephalalgia 2018;38:1773-81.

35. Wang X, Li X, Diao Y, et al. Are Glucose and Insulin Metabolism and Diabetes Associated with Migraine? A Community-Based, Case-Control Study. J Oral Facial Pain Headache 2017;31:240-50.

36. Antonazzo IC, Riise T, Cortese M, et al. Diabetes is associated with decreased migraine risk: A nationwide cohort study. Cephalalgia 2018;38:1759-64.

37. Soares AA, Louçana PMC, Nasi EP, et al. A double- blind, randomized, and placebo-controlled clinical trial with omega-3 polyunsaturated fatty acids (OPFA $₫-3$ ) for the prevention of migraine in chronic migraine patients using amitriptyline. Nutr Neurosci 2018;21:219-23.

38. Ramsden CE, Faurot KR, Zamora D, et al. Targeted alteration of dietary n-3 and n- 6 fatty acids for the treatment of chronic headaches: a randomized trial. Pain 2013;154:2441-51.

39. Maghsoumi-Norouzabad L, Mansoori A, Abed R, et al. Effects of omega-3 fatty acids on the frequency, severity, and duration of migraine attacks: A systematic review and meta-analysis of randomized controlled trials. Nutr Neurosci 2018;21:614-23.

40. Hoffstedt J, Arner P, Hellers G, et al. Variation in adrenergic regulation of lipolysis between omental and 
subcutaneous adipocytes from obese and non-obese men. J Lipid Res 1997;38:795-804.

41. Kokavec A. Effect of sucrose consumption on serum insulin, serum cortisol and insulin sensitivity in migraine: evidence of sex differences. Physiol Behav 2015;142:170-8.

42. Ziegler DK, Hassanein RS, Kodanaz A, et al. Circadian rhythms of plasma cortisol in migraine. J Neurol Neurosurg Psychiatry 1979;42:741-8.

43. Peres MF, Sanchez del Rio M, Seabra ML, et al. Hypothalamic involvement in chronic migraine. J Neurol Neurosurg Psychiatry 2001;71:747-51.

44. Shaw SW, Johnson RH, Keogh HJ. Metabolic changes during glucose tolerance tests in migraine attacks. J Neurol Sci 1977;33:51-9.

45. Lange $\mathrm{P}$, Moreno M, Silvestri E, et al. Fuel economy in food-deprived skeletal muscle: signaling pathways and regulatory mechanisms. FASEB J 2007;21:3431-41.

46. Kim M, Goto T, Yu R, et al. Fish oil intake induces UCP1 upregulation in brown and white adipose tissue via the sympathetic nervous system. Sci Rep 2015;5:18013.

47. Bargut TC, Souza-Mello V, Mandarim-de-Lacerda CA, et al. Fish oil diet modulates epididymal and inguinal adipocyte metabolism in mice. Food Funct 2016;7:1468-76.

48. Bhaskaran S, Unnikrishnan A, Ranjit R, et al. A fish oil diet induces mitochondrial uncoupling and mitochondrial unfolded protein response in epididymal white adipose tissue of mice. Free Radic Biol Med 2017;108:704-14.

49. Saraswathi V, Gao L, Morrow JD, et al. Fish oil increases cholesterol storage in white adipose tissue with concomitant decreases in inflammation, hepatic steatosis, and atherosclerosis in mice. J Nutr 2007;137:1776-82.

50. Hersoug LG, Møller P, Loft S. Role of microbiotaderived lipopolysaccharide in adipose tissue inflammation, adipocyte size and pyroptosis during obesity. Nutr Res Rev 2018;31:153-63.

51. Cinti S, Mitchell G, Barbatelli G, et al. Adipocyte death defines macrophage localization and function in adipose tissue of obese mice and humans. J Lipid Res 2005;46:2347-55.

52. Strissel KJ, Stancheva Z, Miyoshi H, et al. Adipocyte death, adipose tissue remodeling, and obesity complications. Diabetes 2007;56:2910-8.

53. Poret JM, Souza-Smith F, Marcell SJ, et al. High fat diet consumption differentially affects adipose tissue inflammation and adipocyte size in obesity-prone and obesity-resistant rats. Int J Obes (Lond) 2018;42:535-41.

54. Jialal I, Devaraj S. Subcutaneous adipose tissue biology in metabolic syndrome. Horm Mol Biol Clin Investig 2018;33. doi: 10.1515/hmbci-2017-0074.

55. Gollisch KS, Brandauer J, Jessen N, et al. Effects of exercise training on subcutaneous and visceral adipose tissue in normal- and high-fat diet-fed rats. Am J Physiol Endocrinol Metab 2009;297:E495-504.

56. Ding H, Zheng S, Garcia-Ruiz D, et al. Fasting induces a subcutaneous-to-visceral fat switch mediated by microRNA-149-3p and suppression of PRDM16. Nat Commun 2016;7:11533.

57. Li JX, Ke DZ, Yao L, et al. Response of genes involved in lipid metabolism in rat epididymal white adipose tissue to different fasting conditions after long-term fructose consumption. Biochem Biophys Res Commun 2017;484:336-41.

58. Spencer M, Finlin BS, Unal R, et al. Omega-3 fatty acids reduce adipose tissue macrophages in human subjects with insulin resistance. Diabetes 2013;62:1709-17.

59. Sun K, Kusminski CM, Scherer PE. Adipose tissue remodeling and obesity. J Clin Invest 2011;121:2094-101.

60. Harford KA, Reynolds CM, McGillicuddy FC, et al. Fats, inflammation and insulin resistance: insights to the role of macrophage and T-cell accumulation in adipose tissue. Proc Nutr Soc 2011;70:408-17.

61. Goadsby PJ, Edvinsson L, Ekman R. Vasoactive peptide release in the extracerebral circulation of humans during migraine headache. Ann Neurol 1990;28:183-7.

62. Ashina M, Bendtsen L, Jensen R, et al. Evidence for increased plasma levels of calcitonin gene-related peptide in migraine outside of attacks. Pain 2000;86:133-8.

63. Lee MJ, Lee SY, Cho S, et al. Feasibility of serum CGRP measurement as a biomarker of chronic migraine: a critical reappraisal. J Headache Pain 2018;19:53.

64. Spiridonov VK, Tolochko ZS, Korolenko TA. Effect of Transcutaneous Electrical Stimulation of Nerves on Blood Pressure and Blood Content of Neuropeptide CGRP and Nitric Oxide in Hypertensive Rats with Metabolic Disturbances. Bull Exp Biol Med 2019;166:436-9.

65. Lee SH, Tonello R, Im ST, et al. Resolvin D3 controls mouse and human TRPV1-positive neurons and preclinical progression of psoriasis. Theranostics 2020;10:12111-26.

66. Bursać BN, Vasiljević AD, Nestorović NM, et al. Highfructose diet leads to visceral adiposity and hypothalamic leptin resistance in male rats--do glucocorticoids play a role? J Nutr Biochem 2014;25:446-55.

67. Neeb L, Anders L, Euskirchen P, et al. Corticosteroids alter CGRP and melatonin release in cluster headache 
episodes. Cephalalgia 2015;35:317-26.

68. Gram DX, Hansen AJ, Wilken M, et al. Plasma calcitonin gene-related peptide is increased prior to obesity, and sensory nerve desensitization by capsaicin improves oral glucose tolerance in obese Zucker rats. Eur J Endocrinol 2005;153:963-9.

69. Walker CS, Li X, Whiting L, et al. Mice lacking the neuropeptide alpha-calcitonin gene-related peptide are protected against diet-induced obesity. Endocrinology 2010;151:4257-69.

70. Razeghi Jahromi S, Ghorbani Z, Martelletti P, et al. Association of diet and headache. J Headache Pain 2019;20:106.

71. Martins-Oliveira M, Akerman S, Holland PR, et al. Neuroendocrine signaling modulates specific neural networks relevant to migraine. Neurobiol Dis 2017;101:16-26.

72. Bernecker C, Pailer S, Kieslinger P, et al. GLP-2 and leptin are associated with hyperinsulinemia in non-obese female migraineurs. Cephalalgia 2010;30:1366-74.

73. Guldiken B, Guldiken S, Demir M, et al. Low leptin levels in migraine: a case control study. Headache 2008;48:1103-7.

74. Friedman JM, Halaas JL. Leptin and the regulation of body weight in mammals. Nature 1998;395:763-70.

75. Ahima RS, Prabakaran D, Mantzoros C, et al. Role of leptin in the neuroendocrine response to fasting. Nature 1996;382:250-2.

76. Mori TA, Bao DQ, Burke V, et al. Dietary fish as a major component of a weight-loss diet: effect on serum lipids, glucose, and insulin metabolism in overweight hypertensive subjects. Am J Clin Nutr 1999;70:817-25.

77. Caron A, Lee S, Elmquist JK, et al. Leptin and brainadipose crosstalks. Nat Rev Neurosci 2018;19:153-65.

78. Morash B, Li A, Murphy PR, et al. Leptin gene expression in the brain and pituitary gland. Endocrinology 1999; 140:5995-8.

79. Vujovic P, Lakic I, Laketa D, et al. Time-dependent effects of starvation on serum, pituitary and hypothalamic leptin levels in rats. Physiol Res 2011;60:S165-70.

80. Pandit R, Beerens S, Adan RAH. Role of leptin in energy expenditure: the hypothalamic perspective. Am J Physiol Regul Integr Comp Physiol 2017;312:R938-47.

81. Trujillo ME, Scherer PE. Adiponectin--journey from an adipocyte secretory protein to biomarker of the metabolic syndrome. J Intern Med 2005;257:167-75.

82. Nguyen TMD. Adiponectin: Role in Physiology and Pathophysiology. Int J Prev Med 2020;11:136.
83. Neumeier M, Weigert J, Schäffler A, et al. Different effects of adiponectin isoforms in human monocytic cells. J Leukoc Biol 2006;79:803-8.

84. Peterlin BL, Bigal ME, Tepper SJ, et al. Migraine and adiponectin: is there a connection? Cephalalgia 2007;27:435-46.

85. Ramos-Romero S, Molinar-Toribio E, Pérez-Jiménez J, et al. The combined action of omega-3 polyunsaturated fatty acids and grape proanthocyanidins on a rat model of dietinduced metabolic alterations. Food Funct 2016;7:3516-23.

86. Neschen S, Morino K, Rossbacher JC, et al. Fish oil regulates adiponectin secretion by a peroxisome proliferator-activated receptor-gamma-dependent mechanism in mice. Diabetes 2006;55:924-8.

87. Sneddon AA, Rayner DV, Mitchell SE, et al. Dietary supplementation with conjugated linoleic acid plus n-3 polyunsaturated fatty acid increases food intake and brown adipose tissue in rats. Nutrients 2009;1:178-96.

88. Rausch J, Gillespie S, Orchard T, et al. Systematic review of marine-derived omega-3 fatty acid supplementation effects on leptin, adiponectin, and the leptin-toadiponectin ratio. Nutr Res 2021;85:135-52.

89. Choi HM, Doss HM, Kim KS. Multifaceted Physiological Roles of Adiponectin in Inflammation and Diseases. Int J Mol Sci 2020;21:1219.

90. Rizzo MR, Fasano R, Paolisso G. Adiponectin and Cognitive Decline. Int J Mol Sci 2020;21:2010.

91. Cnop M, Havel PJ, Utzschneider KM, et al. Relationship of adiponectin to body fat distribution, insulin sensitivity and plasma lipoproteins: evidence for independent roles of age and sex. Diabetologia 2003;46:459-69.

92. Pellmé F, Smith U, Funahashi T, et al. Circulating adiponectin levels are reduced in nonobese but insulinresistant first-degree relatives of type 2 diabetic patients. Diabetes 2003;52:1182-6.

93. Coope A, Milanski M, Araújo EP, et al. AdipoR1 mediates the anorexigenic and insulin/leptin-like actions of adiponectin in the hypothalamus. FEBS Lett 2008;582:1471-6.

94. Qi Y, Takahashi N, Hileman SM, et al. Adiponectin acts in the brain to decrease body weight. Nat Med 2004;10:524-9.

95. Fang H, Judd RL. Adiponectin Regulation and Function. Compr Physiol 2018;8:1031-63.

96. Mishra D, Richard JE, Maric I, et al. Parabrachial Interleukin-6 Reduces Body Weight and Food Intake and Increases Thermogenesis to Regulate Energy Metabolism. Cell Rep 2019;26:3011-26.e5. 
97. Stenlöf K, Wernstedt I, Fjällman T, et al. Interleukin-6 levels in the central nervous system are negatively correlated with fat mass in overweight/obese subjects. J Clin Endocrinol Metab 2003;88:4379-83.

98. Wallenius K, Wallenius V, Sunter D, et al. Intracerebroventricular interleukin-6 treatment decreases body fat in rats. Biochem Biophys Res Commun 2002;293:560-5.

99. Schöbitz B, Pezeshki G, Pohl T, et al. Soluble interleukin-6 (IL-6) receptor augments central effects of IL-6 in vivo. FASEB J 1995;9:659-64.

100. Thesing CS, Bot M, Milaneschi Y, et al. Omega-3 polyunsaturated fatty acid levels and dysregulations in biological stress systems. Psychoneuroendocrinology 2018;97:206-15

101.De Souza CT, Araujo EP, Bordin S, et al. Consumption of a fat-rich diet activates a proinflammatory response and induces insulin resistance in the hypothalamus. Endocrinology 2005;146:4192-9.

102. Wang X, Ge A, Cheng M, et al. Increased hypothalamic inflammation associated with the susceptibility to obesity in rats exposed to high-fat diet. Exp Diabetes Res 2012;2012:847246.

103.Xu MX, Yu R, Shao LF, et al. Up-regulated fractalkine (FKN) and its receptor CX3CR1 are involved in fructoseinduced neuroinflammation: Suppression by curcumin. Brain Behav Immun 2016;58:69-81.

104. Thaler JP, Yi CX, Schur EA, et al. Obesity is associated with hypothalamic injury in rodents and humans. J Clin Invest 2012;122:153-62.

105. Takizawa T, Qin T, Lopes de Morais A, et al. Noninvasively triggered spreading depolarizations induce a rapid pro-inflammatory response in cerebral cortex. J Cereb Blood Flow Metab 2020;40:1117-31.

106. Fang X, Ge K, Song C, et al. Effects of n-3PUFAs on autophagy and inflammation of hypothalamus and body weight in mice. Biochem Biophys Res Commun 2018;501:927-32.

107.Abdolahi M, Tafakhori A, Togha M, et al. The synergistic effects of $\omega-3$ fatty acids and nano-curcumin supplementation on tumor necrosis factor (TNF)- $\alpha$ gene expression and serum level in migraine patients. Immunogenetics 2017;69:371-8.

108. Mattei G, Padula MS, Rioli G, et al. Metabolic Syndrome, Anxiety and Depression in a Sample of Italian Primary Care Patients. J Nerv Ment Dis 2018;206:316-24.

109. Peres MFP, Mercante JPP, Tobo PR, et al. Anxiety and depression symptoms and migraine: a symptom-based approach research. J Headache Pain 2017;18:37.

110. Gardner A, Boles RG. Beyond the serotonin hypothesis: mitochondria, inflammation and neurodegeneration in major depression and affective spectrum disorders. Prog Neuropsychopharmacol Biol Psychiatry 2011;35:730-43.

111. Winokur A, Maislin G, Phillips JL, et al. Insulin resistance after oral glucose tolerance testing in patients with major depression. Am J Psychiatry 1988;145:325-30.

112.Johnson RJ, Wilson WL, Bland ST, et al. Fructose and Uric Acid as Drivers of a Hyperactive Foraging Response: A Clue to Behavioral Disorders Associated with Impulsivity or Mania? Evol Hum Behav 2021;42:194-203.

113. Barrera CM, Hunter RE, Dunlap WP. Hyperuricemia and locomotor activity in developing rats. Pharmacol Biochem Behav 1989;33:367-9.

114. Sutin AR, Cutler RG, Camandola S, et al. Impulsivity is associated with uric acid: evidence from humans and mice. Biol Psychiatry 2014;75:31-7.

115.Farajdokht F, Babri S, Karimi P, et al. Chronic ghrelin treatment reduced photophobia and anxiety-like behaviors in nitroglycerin- induced migraine: role of pituitary adenylate cyclase-activating polypeptide. Eur J Neurosci 2017;45:763-72.

116. Kiecolt-Glaser JK, Belury MA, Andridge R, et al. Omega-3 supplementation lowers inflammation and anxiety in medical students: a randomized controlled trial. Brain Behav Immun 2011;25:1725-34.

117. Réus GZ, Maciel AL, Abelaira HM, et al. $\omega-3$ and folic acid act against depressive-like behavior and oxidative damage in the brain of rats subjected to early- or late-life stress. Nutrition 2018;53:120-33.

118. Chang JP, Su KP, Mondelli V, et al. Omega-3 Polyunsaturated Fatty Acids in Youths with Attention Deficit Hyperactivity Disorder: a Systematic Review and Meta-Analysis of Clinical Trials and Biological Studies. Neuropsychopharmacology 2018;43:534-45.

119. Demartini C, Greco R, Zanaboni AM, et al. Nitroglycerin as a comparative experimental model of migraine pain: From animal to human and back. Prog Neurobiol 2019;177:15-32.

120. Karsan N, Bose PR, Thompson C, et al. Headache and non-headache symptoms provoked by nitroglycerin in migraineurs: A human pharmacological triggering study. Cephalalgia 2020;40:828-41.

121. Kuroda M, Yoshikawa D, Nishikawa K, et al. Volatile anesthetics inhibit calcitonin gene-related peptide 
receptor-mediated responses in pithed rats and human neuroblastoma cells. J Pharmacol Exp Ther 2004;311:1016-22.

Cite this article as: Barbosa IR, Cunha G, Silva RBM, Freitas RDS, Dagnino APA, Campos MM. Does fructose have a protective role on migraine?-experimental evidence in a rat model of metabolic syndrome under omega-3 supplementation. Ann Transl Med 2022;10(8):435. doi: 10.21037/atm-21-5699
122. Wu X, Lu Y, Dong Y, et al. The inhalation anesthetic isoflurane increases levels of proinflammatory TNF- $\alpha$, IL6, and IL-1 $\beta$. Neurobiol Aging 2012;33:1364-78. 
Supplementary

Table S1 Descriptive statistics showing the variations of the grimace scale AUC values in the following experimental groups: water control (water + saline); HFD control (HFD + saline); migraine-like pain induction (water + NTG); and HFD plus migraine-like pain induction (HFD + NTG); with or without n-3 supplementation (water + n-3; HFD + n-3; n-3 + NTG; HFD + n-3 + NTG)

\begin{tabular}{|c|c|c|c|c|c|c|c|c|}
\hline & \multicolumn{4}{|c|}{ Water } & \multicolumn{4}{|c|}{ HFD } \\
\hline Minimum Values & 0.18 & 5.70 & 0.00 & 3.83 & 0.00 & 3.26 & 0.00 & 5.89 \\
\hline Maximum Values & 0.82 & 7.95 & 2.18 & 7.83 & 1.53 & 7.30 & 2.03 & 7.86 \\
\hline Sum of Totals & 4.58 & 57.09 & 4.00 & 46.94 & 3.17 & 40.67 & 4.95 & 58.91 \\
\hline
\end{tabular}

Data are presented with a number of 7 to 8 rats per group. 